\title{
Measuring metacognitive performance: type 1 performance dependence and test-retest reliability
}

Matthias Guggenmos ${ }^{1 *}$

${ }^{1}$ Charité - Universitätsmedizin Berlin, corporate member of Freie Universität Berlin and Humboldt-Universität zu Berlin, Department of Psychiatry and Neurosciences, Charitéplatz 1, 10117 Berlin, Germany

* Corresponding author: Matthias Guggenmos (mg.corresponding@gmail.com) 


\begin{abstract}
Research on metacognition - thinking about thinking - has grown rapidly and fostered our understanding of human cognition in healthy individuals and clinical populations. Of central importance is the concept of metacognitive performance, which characterizes the capacity of an individual to estimate and report the accuracy of primary (type 1) cognitive processes or actions ensuing from these processes. Arguably one of the biggest challenges for measures of metacognitive performance is their dependency on objective type 1 performance, although more recent methods aim to address this issue. The present work scrutinizes the most popular metacognitive performance measures in terms of two critical characteristics: independence of type 1 performance and test-retest reliability. Analyses of data from the Confidence Database (total $\mathrm{N}=6912$ ) indicate that no current metacognitive performance measure is independent of type 1 performance. The shape of this dependency is largely reproduced by extending current models of metacognition with a source of metacognitive noise. Moreover, the reliability of metacognitive performance measures is highly sensitive to the combination of type 1 performance and trial number. Importantly, trial numbers frequently employed in metacognition research are too low to achieve an acceptable level of test-retest reliability. Among common task characteristics, simultaneous choice and confidence reports most strongly improved reliability. Finally, general recommendations about design choices and analytical remedies for studies investigating metacognitive performance are provided.
\end{abstract}




\section{Introduction}

Being able to introspect about the correctness of thoughts and actions comes with clear benefits both at an individual and a group level. For instance, at an individual level, accurately judging confidence in one's beliefs allows to balance the costs and benefits of possible actions associated with these beliefs (Fleming et al., 2012). At a group level, communicating accurate levels of certainty optimizes collaborative decision-making (Frith, 2008; Bahrami et al., 2012).

Researchers have long aimed to develop measures that accurately capture the performance in such metacognitive judgements. One of the earliest methods is based on the concept of "type 2" receiver operating characteristic (ROC) curves (Clarke et al., 1959; Pollack, 1959). Whereas regular ROC curves contrast the objective probabilities of false alarms and hits, type 2 ROC curves contrast the subjective probabilities of being correct between instances in which those decisions are factually correct ("type 2 hit") and incorrect ("type 2 false alarm"). Thus, while the area under the type 1 ROC curve provides a measure for type 1 sensitivity, the area under the type 2 ROC curve (AUROC2) provides a measure of metacognitive sensitivity (Hosseini and Ferrell, 1982; Critchfield, 1993; Galvin et al., 2003).

A key advantage of AUROC2 over more simple measures such as the correlation between accuracy and confidence (cf. Nelson, 1984) is that $A U R O C 2$ is insensitive to metacognitive biases, i.e. whether an observer generally prefers lower or higher confidence ratings. However, AUROC2 - like correlation-based measures - is dependent on type 1 performance. This can intuitively be understood in terms of type 1 performance influencing the proportion of trials in which an observer has to guess. As even a metacognitively ideal observer cannot predict which decisions, among guessing trials, will be correct, AUROC2 necessarily decreases with an increasing proportion of guessing trials and thus decreasing performance (for a mathematical treatment, see Galvin et al., 2003). Thus, AUROC2 cannot isolate metacognitive performance from type 1 performance.

A solution to this issue was proposed by Maniscalco and Lau $(2012,2014)$ through a measure called meta- $d^{\prime}$. The idea of meta- $d^{\prime}$ is to express metacognitive sensitivity in terms of the type 1 sensitivity that an ideal metacognitive observer would need in order to achieve the observed type 2 hit and false alarm rates. Since meta- $d^{\prime}$ is expressed in units of $d^{\prime}$, it can be directly compared to - and normalized by - type 1 sensitivity. In this way, differences in metacognitive performance that are expected on the basis of type 1 performance differences alone can be mathematically corrected for. If the observer is indeed metacognitively optimal, meta- $d^{\prime}=d^{\prime}$ is expected, whereas values of meta- $d$ ' below $d^{\prime}$ indicate varying degrees of metacognitive suboptimality. As pointed out by Maniscalco and Lau (2012), due to the ratio scaling properties of $d^{\prime}$ measures, this normalisation may be achieved either by subtraction $\left(M_{\text {diff }}=\right.$ meta- $\left.d^{\prime}-d^{\prime}\right)$ or division $\left(M_{\text {ratio }}=\right.$ meta- $\left.d^{\prime} / d^{\prime}\right)$. Fleming and Lau coined the term metacognitive efficiency for these two measures, as they quantify how efficiently observers make use of the available type 1 information for metacognitive judgements (Fleming and Lau, 2014).

Since their inception around 10 years ago, $M_{\text {diff }}$ and in particular $M_{\text {ratio, }}$ have grown in popularity and are now in widespread use in the metacognition community. A frequent use case are 
between-subject designs in which performance levels might differ between participants and/or groups and which thus explicitly require a measures invariant to type 1 performance (Baird et al., 2014; Hauser et al., 2017; Sadeghi et al., 2017; Faivre et al., 2020; Hertz et al., 2020; Nicholson et al., 2020; Ordin et al., 2020; Reyes et al., 2020). Other use cases are within-subject designs in which metacognitive performance is compared between experimental conditions (Maniscalco and Lau, 2015; Odegaard et al., 2018; Shekhar and Rahnev, 2018; Filevich et al., 2020; Konishi et al., 2020; Mei et al., 2020; Ordin and Polyanskaya, 2020) or in which relationships between two domains (e.g. brain and behavior) are assessed (Baird et al., 2013, 2015; McCurdy et al., 2013; Fitzgerald et al., 2017; Samaha and Postle, 2017; Lee et al., 2018; Ye et al., 2019). In both cases, difference in the underlying type 1 performance would be a confounding variable. A particular focus has been the question whether metacognitive performance is a trait-like construct that generalizes across sensory modalities or domains (e.g. memory and perception), with so far mixed results (Baird et al., 2013; McCurdy et al., 2013; Samaha and Postle, 2017; Lee et al., 2018), pointing also to a role of task design (Samaha and Postle, 2017; Lee et al., 2018).

Yet, despite the broad acceptance of metacognitive performance measures based on meta- $d$ ', it is largely unknown whether the claim of type 1 performance invariance holds in practise. Indeed, a recent study demonstrated via simulation that type 1 performance invariance breaks down when assuming that confidence ratings are influenced by additional sources of (metacognitive) noise (Bang et al., 2019). Specifically, according to their simulation, both $M_{\text {ratio }}$ and $M_{\text {diff }}$ increase with increasing levels of sensory noise. To test this prediction, Bang and colleagues additionally performed a behavioral study in which sensory noise of participants was decreased through training over multiple days in a perceptual learning paradigm. Consistent with the model prediction, metacognitive efficiency decreased over the course of the experiment. Irrespective of this particular empirical finding, it seems highly likely that confidence ratings are influenced by additional sources of noise, both during the computation of metacognitive estimates and during report.

A second requirement of quantitative psychological constructs is a sufficient degree of test-retest reliability. All else equal, two measurements of metacognitive performance should give comparable results between a test and a retest session. Indeed, measurement errors for metacognitive performance are a priori expected to be rather high, as they are influenced by measurement errors of both type 1 and type 2 performance. Moreover, type 1 performance by itself is a notoriously noisy measure, as it is derived from a binary variable (correct/incorrect).

The goal of the present work is to shed light on both issues, type 1 performance independence and reliability (test-retest). My approach was two-fold in both cases. In a first step, simulated data was used to study the characteristics of metacognitive performance measures under controlled settings. Second, the same analysis used for simulation was applied to empirical data, making use of the recently released Confidence Database (Rahnev et al., 2020), a continuously growing collaborative repository of confidence datasets (145 at the time of accessing the database). This database comprises a large number of modalities, paradigms and various types of confidence reports and thus provides a powerful dataset to robustly assess measures of metacognition. 
Overall, two measures of metacognitive sensitivity (meta-d' and AUROC2) were assessed and several variants of metacognitive efficiency: $M_{\text {diff, }} M_{\text {ratio }}, M_{\text {ratio }}$ with excluding extreme values, and three regularized variants of $M_{\text {ratio }}$ (bounded, logarithmic, hierarchical $M_{\text {ratio }}$ ). To assess type 1 performance invariance, sensory noise was systematically varied for the simulation-based analysis, whereas the natural variation of type 1 performance was utilized for the empirical analysis of the Confidence Database. To assess test-retest reliability, two artificial sessions of an experiment were generated for simulation-based analysis and split-half subsets of each participant's data were created for the empirical analysis.

The paper is structured in three parts. The first and the second part are concerned with type 1 performance dependency and reliability of metacognitive performance measures, respectively. In the third part, I investigate task characteristics of studies in the Confidence Database that affect the test-retest reliability of metacognitive performance measures.

\section{Results}

Figure 1 introduces the type 1 and type 2 performance measures investigated in this study in terms of their distribution in the Confidence Database. For the creation of these distributions I included participants with at least 400 trials and a certain level of above-chance performance $\left(d^{\prime}>0.5\right)$.

Type 1 performance $d^{\prime}$ shows a peak at an intermediate performance level (corresponding to around $75 \%$ correct responses), which is likely due to staircase procedures which often target intermediate performance levels. The distribution has a positive skew. While the distributions of the two metacognitive sensitivity measures, $A U R O C 2$ and meta- $d$ ', are relatively symmetric and close to a normal distribution, the histogram of $M_{\text {diff }}$ is clearly affected by type 1 performance (the asymmetry is in the opposite direction to $d^{\prime}$, as $M_{\text {diff }}$ involves a subtraction of $d^{\prime}$ ).

By contrast, the distribution of $M_{\text {ratio }}$ is closer to a normal distribution. However, despite the conservative inclusion criteria, $M_{\text {ratio }}$ shows a significant fraction of unrealistically extreme values. $2.4 \%$ of subjects have negative $M_{\text {ratio }}$ values and $4 \%$ have $M_{\text {ratio }}$ values higher than 1.5 . This problem is more severe in studies with fewer trials per subject (e.g. $4 \%$ negative values and $10 \%$ values $>1.5$ in studies with less than 200 trials).

To address the instability of $M_{\text {ratio, }}$ we additionally evaluated a scenario in which extreme values of $M_{\text {ratio }}$ are excluded, as well as three regularization methods. Figure $1 \mathrm{~F}$ shows the distribution of $M_{\text {ratio }}$ values after excluding participants with negative $M_{\text {ratio }}$ values (affecting $2.4 \%$ of the $M_{\text {ratio }}$ values in Figure $1 E$ ) and $M_{\text {ratio }}$ values higher than an upper bound of 1.6 that is symmetric with respect to the median of 0.8 (affecting likewise $2.4 \%$ of $M_{\text {ratio }}$ values). This measure is henceforth referred to as $M_{\text {ratio }}$ (excl.). As a result of excluding these participants, the normality of the $M_{\text {ratio }}$ distribution slightly increases.

The first regularization method, bounding, forces lower and upper bounds for $M_{\text {ratio }}$. To my knowledge, this method has not yet been used in the literature. In the absence of a reference, I thus chose the same bounds of 0 and 1.6 as discussed above, which introduces a noticeable probability mass at both bounds (Figure 1G). A second regularization method, taking the logarithm, was 
suggested by (Fleming and Lau, 2014) to address the occurrence of extreme values and non-normality. However, as Figure $1 \mathrm{H}$ shows, taking the logarithm leads to a heavily asymmetric distribution with a long left tail. If the goal is normality, taking the logarithm is thus not advised. Finally, I tested a hierarchical Bayesian estimation method introduced by (Fleming, 2017) which effectively regularizes extreme values by means of a group prior. The distribution of hierarchical $M_{\text {ratio }}$ values shows a slight positive skew (Figure 1I).
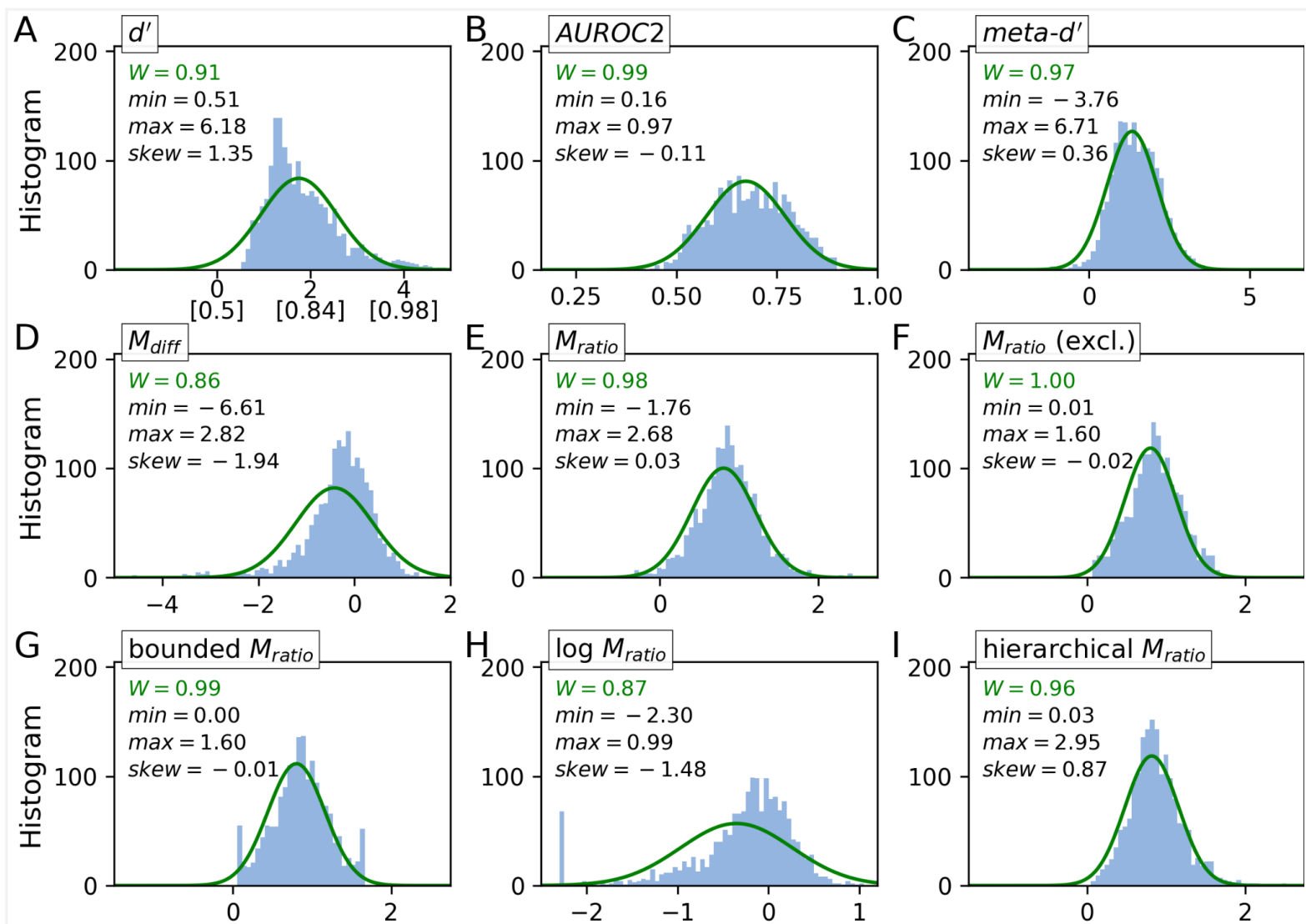

Figure 1. Distributions of $d^{\prime}$ and metacognitive performance measures in the Confidence Database. Only subjects with at least 400 trials were included. A) Type 1 performance measured as $d^{\prime}$. Values in square brackets on the $x$-axis represent proportion correct responses assuming an equal proportion of trials for both stimulus categories. B) Area under the type 2 receiver operating curve (AUROC2). C) Meta-d'. D) $M_{\text {diff }}$ the difference between meta- $d^{\prime}$ and $d^{\prime}$. E) $M_{\text {ration }}$ the ratio of meta- $d^{\prime}$ and $d^{\prime}$. F) Excluding participants with an $M_{\text {ratio }}$ lower than 0 or higher than 1.6. G) Bounded $M_{\text {ratio }}$ with lower bound 0 and a symmetric upper bound of 1.6 (both the mean and the median are close to 0.8). H) Logarithmized $M_{\text {ratio }}$ in which $M_{\text {ratio }}$ values are floored at 0.1 before logarithmization. Note that the normal fit is almost unaffected when excluding the subjects of the lower bound peak. I) Hierarchical $M_{\text {ratio, }}$ based on Bayesian parameter estimation (Fleming, 2017). Green lines indicate fits of a normal distribution (scaled by the histogram amplitude). For each distribution, the W statistic based on a Shapiro-Wilk normality test (higher values indicate higher normality; Shapiro and Wilk, 1965), minimum/maximum values and the Fisher-Pearson coefficient of skewness are provided. 


\section{The relationship between metacognitive performance measures and type 1 performance}

\subsection{Simulation}

In a first step, I simulated the relationship between the investigated set of metacognitive performance measures and type 1 performance. The advantages of simulated data are that they allow precise control over underlying parameters of the generative model, a systematic evaluation across an arbitrary range of relevant variables (including sample size) and high statistical power. At the same time it must be kept in mind that simulated models rely on assumptions about the process of data generation which may deviate from the empirical truth.

Here, I assume that confidence is computed based on the subjective probability of being correct, i.e. using the assumption that observers have a reliable estimate of the stimulus-generating process (in particular an estimate of their own sensory noise). Equipped with such an estimate and using Bayes' rule, an observer is then able to compute a choice probability (method section 4.1; Eq. 3) and can use it to compute confidence (Eq. 4). I assume that reported levels of confidence are subject to metacognitive noise, described by a Beta distribution (Eq. 6). The Beta distribution lends itself for this purpose as it is, by design, a distribution that characterizes the uncertainty of probability estimates. It is thus bounded between 0 and 1 and implicitly avoids other nonsensical values. It can be parameterized with a spread parameter $\sigma_{m}$, which henceforth is referred to as the metacognitive noise parameter. The lower $\sigma_{m}$, the more precise the reported confidence will reflect the choice probability. For the maximum value of $\sigma_{\mathrm{m}}=0.5$, the Beta distribution approaches the uniform distribution and thus confidence reports become random uniform draws from the interval [0;1].

For the purpose of describing the relationship between metacognitive performance and type 1 performance, I simulated data for varying levels of sensory and metacognitive noise. As expected, the two measures of metacognitive sensitivity, AUROC2 (Figure 2A) and meta- $d$ ' (Figure 2B) increase with increasing type 1 performance despite constant metacognitive noise.

Measures of metacognitive efficiency, on the other hand, claim to be invariant with respect to type 1 performance. In the case of $M_{\text {diff, }}$ which corresponds to the subtraction of meta- $d^{\prime}$ and $d^{\prime}$, this is not the case. As shown in Figure $2 \mathrm{C}, M_{\text {diff }}$ shows a clear negative relationship with increasing type 1 performance with the slope becoming more negative with increasing levels of metacognitive noise. This can be understood with an extreme example. Consider an observer that has very high metacognitive noise such that they essentially pick confidence ratings at random. Clearly, for this observer, meta-d' should be equal or close to zero irrespective of type 1 performance. If one thus subtracts type 1 performance $\left(d^{\prime}\right)$ from meta-d', the observed negative relationship is expected. Lower levels of metacognitive noise only attenuate this negative relationship, but even at moderate levels this bias is still substantial.

By contrast, $M_{\text {ratio }}$ is largely stable across different type 1 performance levels (Figure 2D). While for $M_{\text {diff, }}$ the bias is worst for the highest level of metacognitive noise, $M_{\text {ratio }}$ is most unstable for intermediate values of metacognitive noise. In the case of purely random confidence ratings, $M_{\text {ratio }}$ 
stays flat at zero (as it should), simply because the nominator meta-d' is zero. Also for the other extreme - metacognitive noise close to zero $-M_{\text {ratio }}$ yields a stable estimate of 1 . However, for intermediate values of metacognitive noise, $M_{\text {ratio }}$ drops to zero as type 1 performance approaches chance level $\left(d^{\prime}=0\right)$ and also shows a slightly negative slope at higher levels of type 1 performance. The general pattern is very similar when excluding participants with extreme $M_{\text {ratio }}$ values below 0 or above 1.6 (Figure 2E), or for regularized variants of $M_{\text {ratio }}$ (Figures $2 \mathrm{~F}-\mathrm{H}$ ). A minor deviation can be observed for $M_{\text {ratio }}$ (excl.), where a slight positive bias is visible at low levels of type 1 performance and high levels of metacognitive noise.

Overall, while the type 1 performance dependence of AUROC2 and meta- $d^{\prime}$ is well-known and expected, the simulation analyses suggest that the putatively performance-independent measures $M_{\text {diff }}$ and $M_{\text {ratio }}$ do not necessarily hold up to their claim. The performance dependency is particularly clear for $M_{\text {diff }}$, which should not be used as a measure of metacognitive efficiency. The performance dependency of $M_{\text {ratio }}$ is weaker and more complex, depending on the expected range of both type 1 and type 2 performance.
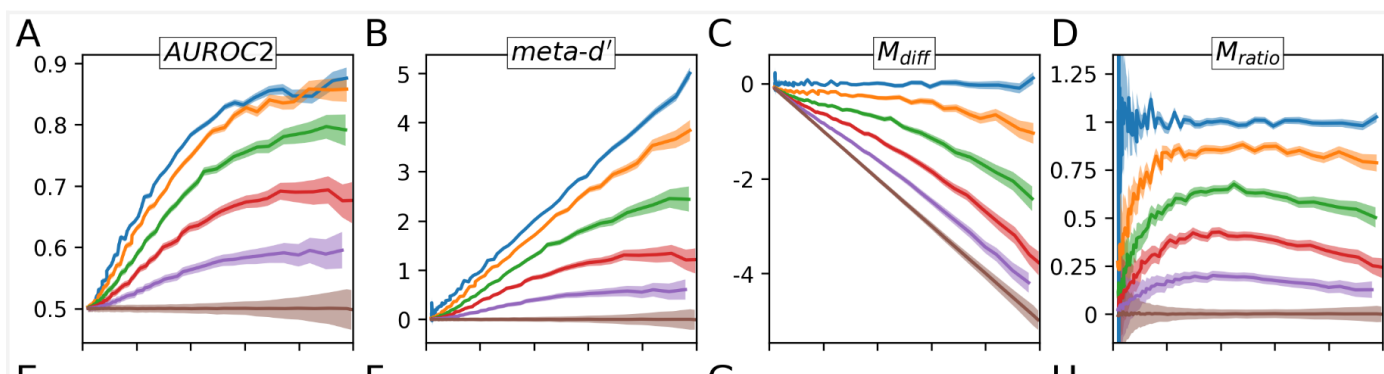

Metacognitive noise $\sigma_{\mathrm{m}}$
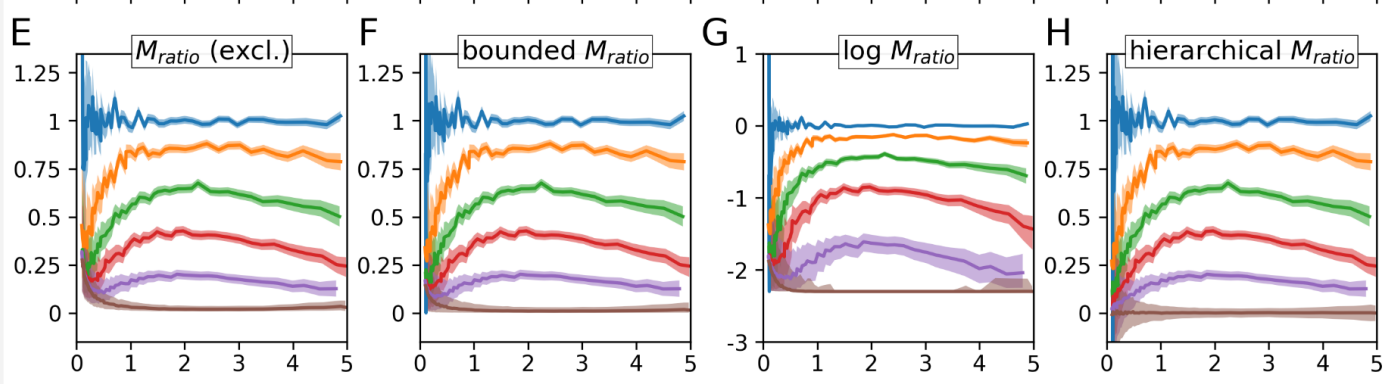

$\begin{array}{ll}- & 0 \\ - & 0.1\end{array}$

Type 1 performance d' [proportion correct]

Figure 2. Simulation: relationship between type 1 (d') and type 2 performance. Shaded areas denote asymmetrical standard deviations. A-B) Both measures of metacognitive sensitivity - AUROC2 and meta-d' increase with increasing type 1 performance. C) $M_{\text {diff }}$ decreases with increasing type 1 performance and more strongly so for increasing levels of metacognitive noise. D) $M_{\text {ratio }}$ is more stable across different performance levels compared to $M_{\text {diff, }}$ but is likewise not independent of type 1 performance in the simulation. An initial pronounced increase at low-levels of performance is followed by a slight decrease starting from $d^{\prime}$ values of around 2 (corresponding to a performance of around 80\% correct). E) Excluding participants with an $M_{\text {ratio }}$ lower than 0 or higher than 1.6. F-H) In the simulation, regularization of $M_{\text {ratio }}$ has no noticeable effect on its dependency on type 1 performance.

\subsection{Empirical data}

In a next step, I evaluated the type 1 performance dependency of metacognitive efficiency measures on empirical data of the Confidence Database, which provides high statistical sample power across a variety of tasks and modalities (Rahnev et al., 2020). I did not test the two measures of 
metacognitive sensitivity, AUROC2 and meta-d', as they are a priori expected to be dependent on type 1 performance (also confirmed in the simulation).

To test for a linear relationship between type 1 performance and each metacognitive efficiency measure, mixed linear models were used with study as a grouping variable and modality (cognitive, memory, motor perception, mixed) as an additional control variable. Moreover, each participants' data were split into two interleaved halves, such that the metacognitive performance measures were computed on a different subset of the data than the type 1 performance measure $d^{\prime}$. This ensured that the measurement noise associated with the estimation of $d^{\prime}$ per se and the measurement noise of the $d^{\prime}$ that enters the computation of metacognitive efficiency measures (either via subtraction or division) were independent, thus preventing spurious correlations.

In agreement with the simulation, $M_{\text {diff }}$ shows a strong negative relationship with type 1 performance (beta \pm SEM $=-0.17 \pm 0.02, p<0.001$; Figure $3 \mathrm{~A}$ ). Thus, also empirically $M_{\text {diff }}$ is not independent of type 1 performance and should thus not be used as a measure of metacognitive efficiency.

$M_{\text {ratio }}$ (without regularization) likewise shows a similar pattern as in the simulation. $\ln$ particular, $M_{\text {ratio }}$ shows a slight but steady decrease with increasing type 1 performance (beta $=-0.08 \pm 0.03$, $p=0.010$ ). As in the simulation, bounding and exclusion of extreme $M_{\text {ratio }}$ values had only moderate effects on the type 1 performance dependency. In both cases, the negative relationship with d' was numerically weaker, but still trendwise significant (bounded $M_{\text {ratio }}$ : beta $=-0.07 \pm 0.04, p=0.063$; $M_{\text {ratio }}$ with exclusion: beta $=-0.08 \pm 0.04, p=0.072$ ). Of note, when pooling participants across studies in a simple regression analysis, the statistical evidence for this negative relationship was stronger (cf. Figures 3C and 3D).

By contrast, the hierarchical estimation and the logarithmization of $M_{\text {ratio }}$ showed an effect with respect to the type 1 performance dependency. While the negative relationship was even more pronounced for the hierarchical $M_{\text {ratio }}$ (beta $=-0.19 \pm 0.05, p<0.001$; Figure 3F), compared to the regular $M_{\text {ratio, }}$ it disappeared for the logarithmized $M_{\text {ratio }}$ (beta $=0.01 \pm 0.02, p=0.581$ ). Of note, when pooling across participants, there was still a negative rank order correlation between the logarithmized $M_{\text {ratio }}$ and $d^{\prime}\left(r_{\mathrm{s}}=-0.073, \mathrm{p}=0.003\right.$; Figure $\left.3 \mathrm{E}\right)$. More data are thus necessary to conclude with certainty that the relationship is indeed absent.

While the empirical data thus largely reproduced the decrease of $M_{\text {ratio }}$ with higher type 1 performance in the simulation, the initial increase of $M_{\text {ratio }}$ at low levels of type 1 performance is much less evident. The regular $M_{\text {ratio }}$ shows a tendency in this direction (Figure 1B), but the scarcity of participants with very low type 1 performance levels prevents a meaningful statistical analysis of this effect. When excluding $M_{\text {ratio }}$ values with extreme values (Figure 1C), this tendency disappears entirely; however, this might be due to the positive bias at low type 1 performance levels observed in the simulation (cf. Figure 2E).

By and large, the results based on the Confidence Database confirm the results of the simulation: a strong negative type 1 performance dependency for $M_{\text {diff }}$ and a weaker, possibly more complex 
dependency of $M_{\text {ratio }}$ and its regularized variants, with a general trend towards lower $M_{\text {ratio }}$ values with higher d'.
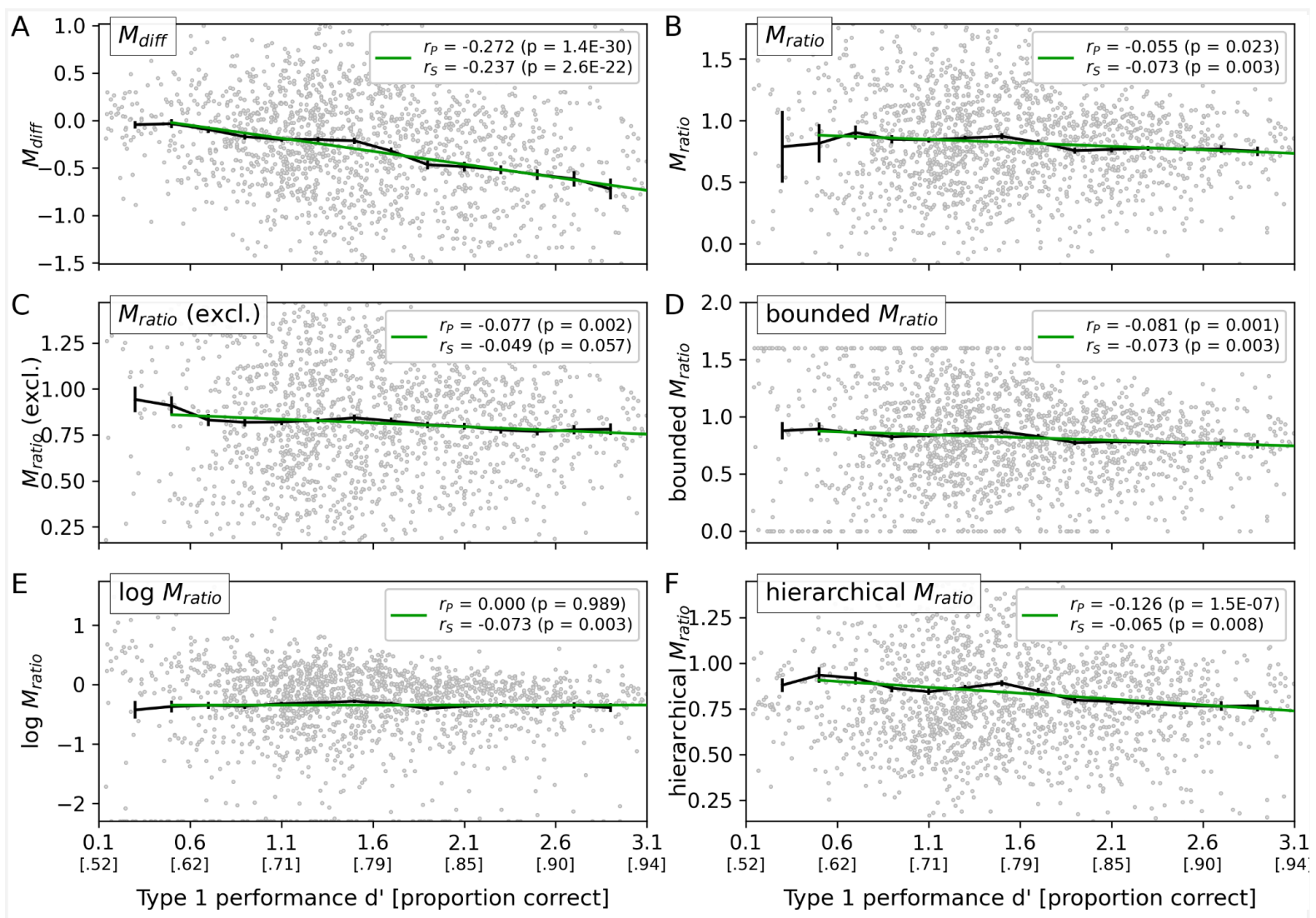

Figure 3. Relationship between type 1 performance $\left(d^{\prime}\right)$ and metacognitive efficiency measures in the Confidence Database. A) Like in the simulation, $M_{\text {diff }}$ shows a clear negative relationship with $d^{\prime}$. B) $M_{\text {ratio }}$ likewise shows a similar pattern as the simulation: a slight initial increase is followed by a decrease with increasing $d^{\prime}$. C) Excluding participants with $M_{\text {ratio }}$ values lower than 0 or higher than 1.6. D-F) Regularized variants of $M_{\text {ratio }}$ : bounding, logarithmic transformation and hierarchical estimation (Fleming, 2017). For all methods except the bounded $M_{\text {ration }}$ the range of the y axis was restricted to the $95 \%$ of data points which were closest to the overall median. Black error bars indicate the mean and the SEM of bins centered at the position of the errorbar and extending $\Delta d^{\prime} \pm 0.2$ left and right of the center. Green lines show the linear regression line to all data points that match the inclusion criterion of $d^{\prime}>0.5$.

\section{Test-retest reliability of metacognitive performance measures}

\subsection{Simulation}

Test-retest reliability is a critical hallmark of any psychological construct and little to nothing is currently known about the reliability of metacognitive performance measures. As for the type 1 performance dependency, I first set out to evaluate the reliability of these measures under the controlled settings of a simulation.

To this aim, I systematically varied the number of trials per subject and the average performance level. For each number of trials and performance level, two artificial experimental sessions were simulated for 100 subjects, referred to as test and retest session, which allowed quantifying the test-retest reliability. Metacognitive noise of each subject was drawn from a uniform distribution 
covering the entire range from an ideal metacognitive observer $\left(\sigma_{m}=0\right)$ to a metacognitively blind observer $\left(\sigma_{\mathrm{m}}=0.5\right)$ which effectively chooses confidence ratings at random.

Two measures of reliability were employed: Pearson correlation and the normalized mean absolute error (NMAE). Whereas the Pearson correlation indicates whether the pattern of results across participants is similar between two measurements, the NMAE provides information about absolute measurement errors. To allow a comparison between metacognitive performance measures, the NMAE normalizes the mean absolute error between the test and retest session values by the mean absolute error between each individual value and the average in the other session. A NMAE of e.g. 0.5 thus indicates that the average difference between test and retest values is half of the average distance between the values and the average of the other session.

For the reliability analysis, I focus on the $M_{\text {ratio }}$ and its regularized variants, as all other metacognitive performance measures are strongly dependent on type 1 performance. This makes it impossible to dissociate whether the reliability is driven by the consistency of type 1 or type 2 performance. For completeness, the reliability of other metacognitive performance measures as well as type 1 performance is provided in supplementary Figure S1.

As expected, the reliability of $M_{\text {ratio }}$ increases with the number of trials per subject, i.e. increasing correlation and decreasing NMAE (Figure 4). Likewise, the reliability increases with increasing type 1 performance. Intuitively, this is because the precision of metacognitive performance estimates naturally increases as the proportion of guessing trials decreases. In contrast, as type 1 performance approaches chance level, there is a dramatic drop in reliability. For instance, at 250 trials and $60 \%$ correct responses, the Pearson reliability is at only $r=0.2$.

To put the overall level of reliability into context, it is worth contrasting it with the reliability of type 1 performance. The Pearson reliability of type 1 performance is close to 1 already at around 250 trials (supplementary Figure S1A) - despite the fact that it is based on noisy binary values (correct / incorrect). In comparison, the reliability of $M_{\text {ratio }}$ is substantially lower in this trial range, not least due to the fact that it is based on a combination of two noisy variables - type 1 and type 2 responses.

Excluding extreme values of $M_{\text {ratio }}$ (Figures $4 \mathrm{~B}$ and $4 \mathrm{~F}$ ) has mainly an effect on the NMAE at low performance levels and low trial numbers. This is not surprising given the fact that these are the conditions that typically produce extreme $M_{\text {ratio }}$ values. Of note, the Pearson correlation appears to be minimally higher for the $M_{\text {ratio }}$ without exclusion, at least under some conditions. A potential explanation is that extreme values introduce larger variance which might have a slight net positive effect on the Pearson correlation.

Can regularization improve the reliability? Taking the logarithm of the $M_{\text {ratio }}$ improves the Pearson reliability only for lower levels of type 1 performance or at lower trial numbers and thus only when $M_{\text {ratio }}$ is expected to be unstable. At typical performance levels ( $>70 \%$ correct) and trial numbers, the simulation suggests that test-retest reliability can even be worse than the original $M_{\text {ratio }}$. By contrast, bounding values of $M_{\text {ratio }}$ improves the Pearson correlation across simulation parameters. Again, improvements are largest at lower levels of type 1 performance and at a lower number of trials. This analysis suggests that - in terms of test-retest reliability - bounding should be preferred over taking 
the logarithm (see also supplementary Figure S2, for a direct comparison of regularization/exclusion methods). Finally, the reliability of the hierarchical $M_{\text {ratio }}$ is highly similar to the bounded $M_{\text {ratio }}$ with the exception of high type 1 performance levels at low trial numbers: here, the hierarchical $M_{\text {ratio }}$ clearly outperforms all other measures.

Overall, I conclude that the reliability of $M_{\text {ratio }}$ will be poor in many realistic scenarios. Studies investigating metacognitive efficiency should thus carefully consider the number of trials and the targeted type 1 performance level, both of which are factors that substantially affect reliability. Regularization methods improve the reliability to a certain degree and are thus generally recommended. Between regularization methods, the simulation shows comparable improvements of the bounded and hierarchical $M_{\text {ratio }}$ in terms of Pearson reliability, while the logarithmic $M_{\text {ratio }}$ performs worse and can even lead to a decrease of Pearson reliability.

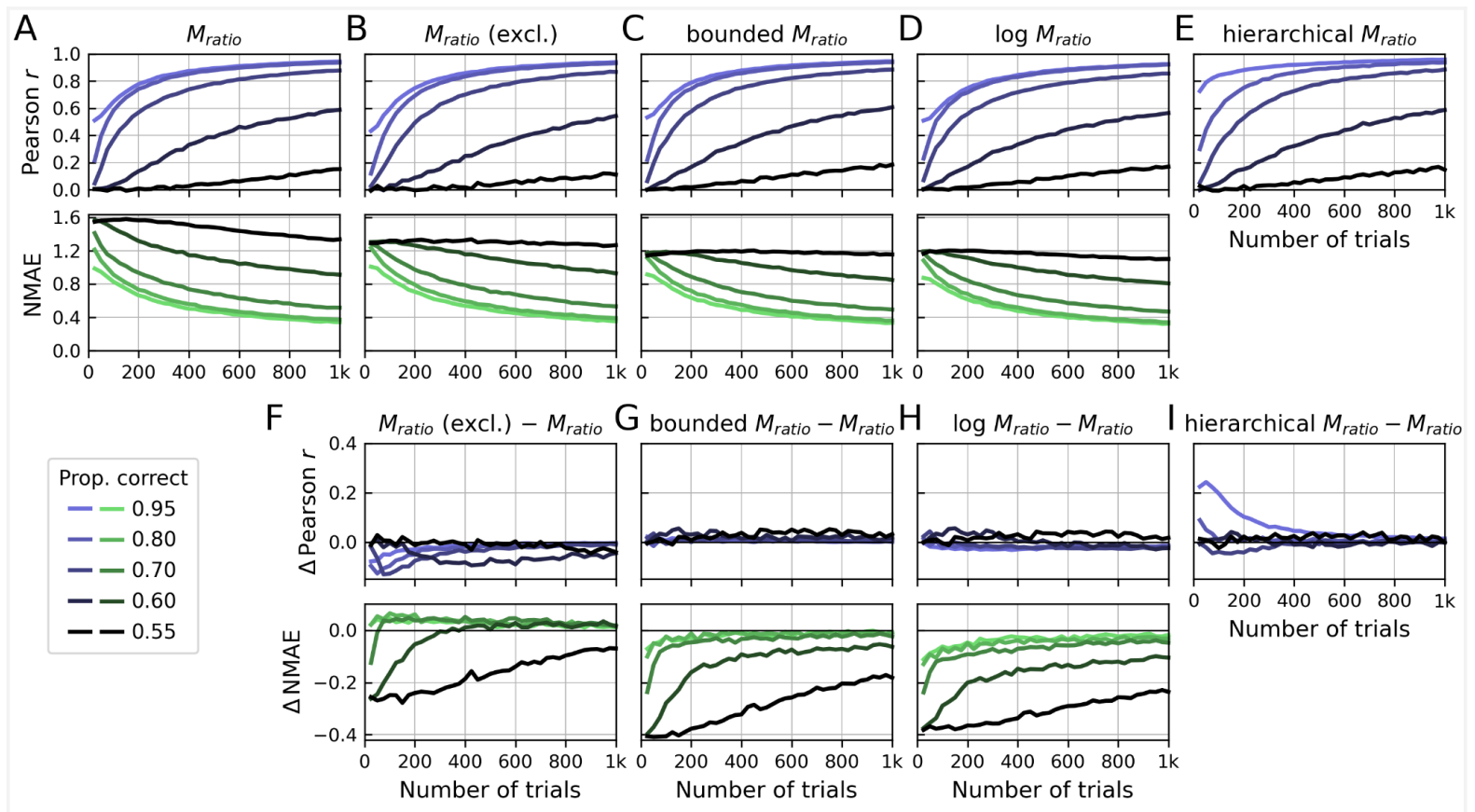

Figure 4. Simulation: test-retest reliability of metacognitive performance measures. For each number of trials and performance level, two hypothetical experimental sessions of 100 subjects were simulated, corresponding to test and retest. This procedure was repeated for $\mathrm{X}$ iterations and averages were computed across iterations. Metacognitive performance measures $\left(M_{\text {ratio }}, M_{\text {ratio }}\right.$ (excl.), bounded $M_{\text {ratio, }} \log M_{\text {ratio }}$, hierarchical $\left.M_{\text {ratio }}\right)$ were computed for each session separately. Reliability was quantified either by means of the Pearson correlation or the normalized mean absolute error (NMAE). Note that the NMAE is not valid for the hierarchical $M_{\text {ratio }}$ (see method section 3) and is thus not shown. A-E) Pearson correlation and the NMAE quantify the reliability of metacognitive performance measures between test and retest. Note that exceedingly extreme value of the regular $M_{\text {ratio }}\left(\left|M_{\text {ratio }}\right|>10\right)$ were excluded, as they caused some instability in the analysis. F-I) Differences in test-retest reliability between measures. 


\subsection{Empirical data}

To assess the test-retest reliability of metacognitive performance measures on empirical data, the data of each subject were divided in two artificial subsets through an interleaved splitting procedure. As the simulation indicated that the trial number per subject is a critical quantity for the reliability of metacognitive performance measures, the studies of the Confidence Database were binned according to the split-half number of trials per subject (1st bin: 0-200 trials, ... 5th bin: 800-1000 trials). Since there was some variance of trial numbers within studies, I excluded participants whose trial numbers were outside the assigned bin of a study. As the large majority of studies have intermediate performance levels ( $85 \%$ of the studies have an average proportion correct between 0.65 and 0.85 ), the studies were not split up according to performance. Importantly, the performance is quite similar for all trial number bins (Figure 3A, black line). As in the simulation, the focus was on $M_{\text {ratio }}$ and its regularized variants (for other measures see supplementary Figure S3).

Without regularization and at moderate trial numbers $(<400)$, the Pearson reliability of $M_{\text {ratio }}$ is quite poor at $r \leq 0.6$ and the NMAE is $\geq 1$, indicating that the $M_{\text {ratio }}$ of a subject at test is, on average, not closer to the same subject's $M_{\text {ratio }}$ at retest than it is to the mean of all subjects (Figure $3 \mathrm{~A}$ ). The Pearson correlation increases to around 0.7 and 0.85 for trial numbers between $400-600$ and 600-800, respectively, and the NMAE drops below 1 . Overall, 400 appears as a sensible minimum recommended trial number for studies using the regular $M_{\text {ratio }}$.

Does regularization improve the reliability of $M_{\text {ratio }}$ ? At low to moderate trial numbers, bounding and logarithmization of $M_{\text {ratio }}$ values leads to slight improvements over the regular $M_{\text {ratio }}$ in terms of NMAE reliability (Figures 3B to $3 \mathrm{G}$ ). The Pearson reliability is largely unaffected by regularization and also does not exhibit the slight advantage of the hierarchical $M_{\text {ratio }}$ observed for high type 1 performance levels in the simulation. There are no detectable differences between the regularization methods (more data would be necessary). Similar to the simulation, excluding $M_{\text {ratio }}$ values slightly decreased the test-retest reliability (Figure $5 \mathrm{~F}$, upper panel). An advantage in terms of NMAE reliability was not observed (Figure 5F, lower panel).

As to be expected, the benefit of regularization vanishes entirely at larger trial numbers. For studies with around 600 trials or more, there is no longer an improvement of NMAE reliability for any regularization method. This suggests that studies that are powered with 600 trials and more per subject can quite safely omit regularization. 


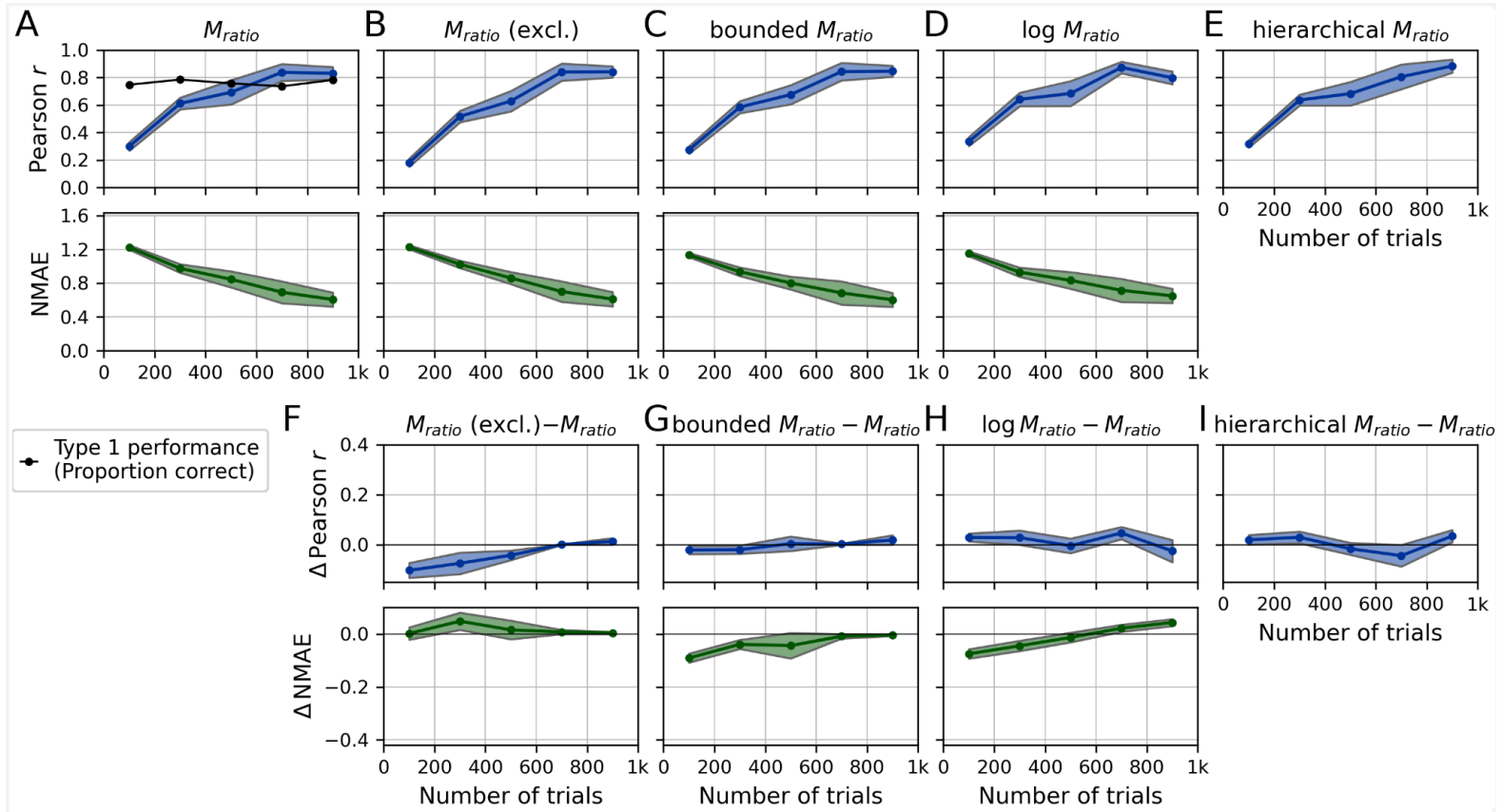

Figure 5. Test-retest reliability of metacognitive performance measures in the Confidence database. Test-retest reliability is computed either as the Pearson correlation coefficient or the normalized mean absolute error (NMAE) between test and retest trials. The $x$-axis denotes the average split-half number of trials for the Confidence Database studies (bins of 200 from 0-1000 trials), i.e. the number of trials per test and retest. In this way, the expected reliability for other/new studies with overall $\mathrm{N}$ trials can simply be obtained by looking up the reliability for $\mathrm{x}=\mathrm{N}$. The accuracy (proportion correct) is relatively constant across bins and thus likely not a confounder. Note that the number of samples refers to the split-half test and retest datasets, hence the total number of samples per subject is twice as high. Shaded areas indicate standard errors across studies. A-E) Test-retest reliability of $M_{\text {ratio }}, M_{\text {ratio }}$ (excl.) and regularized variants. For convenience, type 1 performance is plotted in panel A, given as proportion correct responses. Note that the NMAE is not valid for the hierarchical $M_{\text {ratio }}$ (see method section 3) and is thus not shown. F-I) Differences in test-retest reliability between $M_{\text {ratio }}$ and $M_{\text {ratio }}$ (excl.) / regularized variants of $M_{\text {ratio }}$.

\section{Task characteristics affecting the reliability of metacognitive performance} measures

As shown in the previous section, the number of trials and type 1 performance constrain the expected reliability of $M_{\text {ratio }}$. Both factors must be carefully chosen to achieve an acceptable level of measurement reliability. In this final section I was interested in which other study and task characteristics affect reliability of measured $M_{\text {ratio }}$ values. Specifically, I was interested in the following five task characteristics: 1) the number of available confidence ratings; 2) whether ratings are continuous or not; 3 ) whether confidence ratings are provided simultaneously with type 1 choices; 4) whether feedback is provided; and 5) whether there was an online staircase procedure. I focused on the reliability of regular $M_{\text {ratio }}$ without regularization and therefore selected only studies with at least 400 trials, in line with the recommendations of the previous sections.

The test-retest reliability of $M_{\text {ratio }}$ was computed separately for each study and defined a mixed linear model with the test-retest reliability as the dependent variable and the above task characteristics as the independent variables of interest. Additional control variables were the number of subjects, the number of trials and study-specific averages of type 1 performance $\left(d^{\prime}\right)$, confidence, $M_{\text {ratio }}$ as well as 
modality (categorical predictor: cognitive, motor, perception, mixed or memory). The grouping variable of the mixed model was 'study id', i.e. a unique identifier for each study.

Confirming the previous analyses, type 1 performance and the number of trials strongly predict the test-retest reliability of $M_{\text {ratio }}$ (Figure 4). Among the variables of interest, simultaneous choice and confidence responses and the number of available ratings were associated with higher reliability (only bivariate correlation) and, somewhat surprisingly, external feedback was associated with slightly lower reliability.

The positive effect of simultaneous choice and confidence ratings on the reliability of $M_{\text {ratio }}$ was strong. While the average test-retest correlation is $r=0.83$ when responses are provided simultaneously, it drops to $r=0.51$ when confidence ratings are provided in a separate response after the type 1 choice. Online staircasing showed a trendwise association with lower reliability, which may be interesting in reference to the ongoing discussion about the interaction of staircase procedures and $M_{\text {ratio }}$ (Rahnev and Fleming, 2019).

It is notable that test-retest reliability was unaffected by whether confidence ratings were given on a discrete or continuous scale, although a higher number of available confidence levels slightly improved the Pearson reliability.

The strongest and most robust conclusion from this analysis is the clear benefit of simultaneous type 1 and type 2 responses on the test-retest reliability of $M_{\text {ratio }}$.
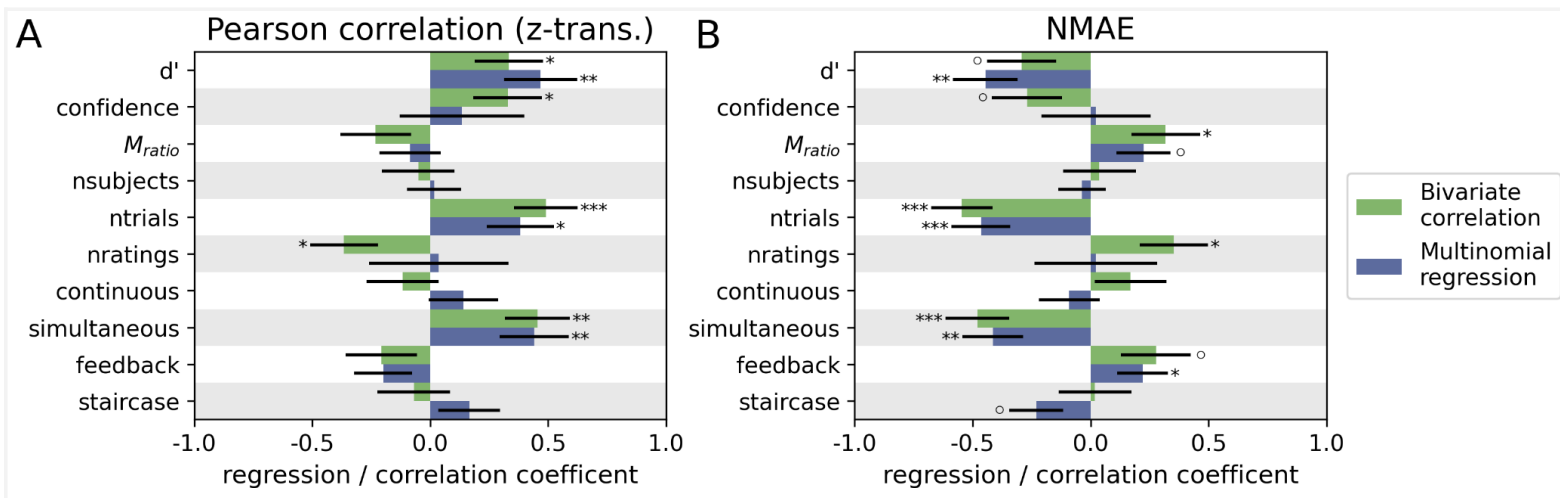

Figure 6. Study characteristics affecting the test-retest reliability of $\boldsymbol{M}_{\text {ratio }}$. Green bars indicate the bivariate correlation coefficient between the predictor and the measure of reliability; blue bars show the regression coefficient of a linear model including all predictors. A) Test-retest reliability measured with the Pearson correlation coefficient. Positive values indicate improvements in reliability. B) Test-retest reliability measured with the NMAE. Negative values indicate improvements in reliability.

Asterisks: ${ }^{\circ} p<0.1, * p<0.05, * * p<0.01,{ }^{* * *} p<0.01$

\section{Discussion}

In the current work I investigated the type 1 performance independence and reliability of metacognitive performance measures. I showed that even measures of metacognitive efficiency, i.e. measures that normalize for type 1 performance, are not necessarily independent of type 1 performance. This dependency is much weaker and thus possibly tolerable for $M_{\text {ratio }}$ compared to 
$M_{\text {diff }}$. The analyses of test-retest reliability showed that $M_{\text {ratio }}$ has quite poor test-retest reliability and standard trial numbers; moreover, the reliability analyses provide guidance and recommendations for regularization methods, trial numbers and task design.

\section{All current measures of metacognitive performance are dependent on type 1 performance}

A quintessential aspect for measures of metacognitive performance is to what degree a measure isolates type 2 from type 1 performance. The intricacy of the tight relationship between type 1 and type 2 has been long known and has led to the development of meta-d' (Rounis et al., 2010; Maniscalco and Lau, 2012, 2014) and two proposals for metacognitive performance measures that explicitly normalize for type 1 performance $\left(M_{\text {diff }}\right.$ and $\left.M_{\text {ratio }}\right)$. Here, I showed through simulation and application to empirical data, that $M_{\text {diff }}$ is heavily biased by type 1 performance and thus should not be used if independence of type 1 performance is important. Specifically, $M_{\text {diff }}$ decreases with increasing type 1 performance and this negative relationship becomes stronger with increasing metacognitive noise.

While $M_{\text {ratio }}$ is clearly more stable across different levels of type 1 performance, it may not be entirely independent. Also here, simulation and application to empirical data show agreement and indicate a relationship between type 1 performance and $M_{\text {ratio }}$ that can be described as an inverted U-shape: as type 1 performance approaches chance level, $M_{\text {ratio }}$ approaches zero; with increasing type 1 performance, $M_{\text {ratio }}$ shows a slight but steady decrease. Of note, this pattern is independent of the number of trials: the simulation used a very large number of 10,000 trials and the pattern did not change when the number of trials was either in- or decreased.

A limitation of simulation-based analyses is that any result could be conditional on the chosen model and the relationship between $M_{\text {ratio }}$ and type 1 performance may be of a different kind for the unknown ground truth model. While it is a good sign that the simulation reproduced the general pattern of the empirical data, it is not a proof. This is less of an issue for the conclusions about $M_{\text {diff }}$ since its type 1 performance dependency can be logically explained and its empirical negative relationship with type 1 performance was so strong that a confirmation by simulation is less important. However, for $M_{\text {ratio }}$ neither of these arguments can be used. In an exploratory analysis I therefore probed the robustness of the simulation result for $M_{\text {ratio }}$ by testing additional metacognitive noise distributions. As shown in Supplementary Figure S4, the results were highly similar to the Beta distribution used in the main simulation. Future studies could test the type 1 performance dependency for different model architectures as well.

An additional aspect to consider is that there might be a true relationship between type 1 performance and metacognitive ability which is not due to a measurement confound. For instance, there could be a true positive relationship reflecting the general observation that cognitive abilities correlate across domains (Carroll, 1993). In this view, the observed negative correlation of the measures might factually be stronger and is masked to some degree by the general positive relationship in the population. Support for this view comes from a recent large-scale model-based analysis of the Dunning-Kruger effect, which concluded that low-performers (in the type 1 task) also tend to have more noisy representations of their type 1 accuracy (Jansen et al., 2021; see also Mazor 
and Fleming, 2021). Alternatively, there could be a true negative relationship because of a potential trade-off in allocating cognitive resources to either the type 1 or the type 2 task. While this highlights the difficulty to conclusively prove the non-independence of metacognitive performance measures and type 1 performance, the burden of proof certainly lies on the side of those claiming independence.

\section{Possible solutions to address the dependency on type 1 performance}

While the type 1 performance dependency of $M_{\text {ratio }}$ may be regarded as relatively subtle, it can nevertheless be an issue, especially as higher and higher sample sizes are collected in online experiments or collaborative research projects. Large sample sizes will be sensitive even to small type 1 performance confounds and lead to artifactual results.

An obvious solution to this issue are online staircase procedures which continuously adjust stimulus difficulty towards a constant target type 1 performance level (e.g. Rouault et al., 2018). However, staircase procedures introduce a different issue. As noted by Rahnev and Fleming (2019), a mix of stimulus difficulty levels can artificially inflate estimates of metacognitive performance relative to a design with a single difficulty level. In brief, the issue is that it is easier for subjects to assign high confidence to correct choices and low confidence to incorrect choices when the experiment itself contains objectively easier and harder trials. This might not be a problem, if the staircase-induced inflation would be a constant shift that cancels out in a comparison between conditions. However, there is no guarantee that the mix of easier and hard trials is identical for different subjects and thus also no guarantee that the shift is constant. For instance, relative to control subjects, a group of patients might show stronger fluctuations of concentration, which could lead to larger adjustments in the staircase procedure. Larger differences in objective stimulus difficulty in turn could lead to an inflated estimate of metacognitive performance. For this reason, Rahnev and Fleming (2019) generally recommend a single difficulty level, which however leads back to the problem of type 1 performance confounds. Offline staircase procedures can alleviate the issue to a certain degree. However, because offline staircase procedures are notoriously imprecise (García-Pérez, 1998) and learning will often continue throughout the main experiment (not necessarily the same in different groups), it is often hard to equate performance reliably with offline staircase procedures.

At present, there is thus no all-purpose solution. In general I recommend to include confounding factors in any analysis of metacognitive performance, i.e. type 1 performance in single-difficulty-level designs and staircase variability (Rahnev and Fleming, 2019) in designs with an online staircase. A specific issue when controlling for type 1 performance is that $d^{\prime}$ effectively would enter both sides of the regression equation, as it is also contained in $M_{\text {ratio }}=$ meta- $d^{\prime} / d^{\prime}$. In this case I recommend using a cross-validated regression approach, such that $d^{\prime}$ in the DV and IV are computed on independent data (as in the analysis in Figure 3). While doing so, one should be aware of the fact that controlling for type 1 performance might remove true interindividual variability in metacognitive performance when type 1 performance and metacognitive ability factually correlate in the population (as noted above). 
The test-retest reliability of $M_{\text {ratio }}$ is strongly affected by type 1 performance and the number of trials

Most studies in metacognition research aim for intermediate type 1 performance levels to introduce a sufficient degree of variance in confidence ratings. The simulation analyses showed that, in terms of test-retest reliability, it makes all the difference whether type 1 performance is at $60 \%, 70 \%$ or $80 \%$ correct responses. At $60 \%$ correct responses or below, the Pearson reliability of $M_{\text {ratio }}$ is very poor even at decent trial numbers of around $400-600(r \approx 0.4)$. By contrast, at $80 \%$ correct responses the Pearson reliability is around $r \approx 0.8$ for $400-600$ trials and thus already substantially better. In the absence of other constraints I thus recommend a performance level of approximately $80 \%$ correct responses for studies investigating metacognitive efficiency.

As for any noisy measure, the test-retest reliability of $M_{\text {ratio }}$ is affected by the number of trials measured for a subject. In general, the number of trials should be higher for lower levels of type 1 performance. For instance, if type 1 performance is at $60 \%$ correct responses around 1000 trials are required according to the simulations to even achieve a Pearson reliability of $r=0.6$. The analysis of the Confidence database, which provides a cross section of trial numbers used by studies in the research field, suggests a minimum recommended trial number of 400 trials for studies measuring metacognitive performance.

\section{Regularization can boost the test-retest reliability of $M_{\text {ratio }}$}

To address the instability of $M_{\text {ration }}$ I evaluated three different methods of regularization (bounded, logarithmic and hierarchical $M_{\text {ratio }}$ ) and tested their effects on test-retest reliability. With the exception of the logarithmic $M_{\text {ration }}$ all regularization methods consistently improved the test-retest reliability. In general, regularization improved the NMAE much stronger than the Pearson correlation. This is not surprising as the NMAE is naturally improved when extreme values are tamed by regularization. Nevertheless, regularization also introduced a slight but significant advantage with respect to the Pearson reliability in both simulated and empirical data. The benefits of regularization vanished as the number of trials increased. For studies with 600 trials or more in the Confidence Database, the reliability was almost indistinguishable for $M_{\text {ratio }}$ with and without regularization.

For studies measuring below 600 trials per subject, I recommend using some form of regularization. Given the heavily asymmetric distribution of $M_{\text {ratio }}$ values following a logarithmic transformation, I advise against this method.

The hierarchical $M_{\text {ratio }}$ can likewise be regarded as a form of regularization and performed well in terms of test-retest reliability with a pronounced advantage relative to other regularization methods for high type 1 performance levels. Nevertheless, its hierarchical estimation approach is not without problems. Hierarchical $M_{\text {ratio }}$ values cannot be compared between studies (when fitted separately) as they depend on the study-specific group average. In within-subject designs, the correlation/variance structure has to be specified with great care to enable valid inferences. For instance, to my knowledge there is currently no available solution for mixed designs with both within- and between-subject factors. However, it should be noted that I used the hierarchical $\mathrm{M}_{\text {ratio }}$ as a means to 
regularize single-subject estimates, whereas its main original aim was allowing for an accurate inference on group-level parameters when individual trial numbers are limited. Although group-level measures are beyond the scope of this, given their increased popularity (e.g., Harrison et al., 2020), future studies should assess the reliability of these measures as well.

In terms of regularization I thus recommend a simple bounding method along the lines suggested in this work. While this method naturally introduces edge densities in the resulting distributions, these edge cases should be relatively rare for reasonable choices of type 1 performance and the number of trials. Applied to the data of the Confidence database, the distribution resulting from bounding showed a high degree of normality for the bounds applied (lower bound 0 and upper bound 1.6). The advantage compared to functional transformations such as the logarithmic transformation is that the absolute $M_{\text {ratio }}$ values resulting from bounding are still in the original scale and thus interpretable.

\section{Task characteristics that improve the test-retest reliability of $M_{\text {ratio }}$}

I argue that the test-retest reliability of metacognitive performance measures can be used as a quality measure for the confidence ratings obtained in a study. In brief, the rationale is that more precise confidence ratings will improve the measurement reliability of the psychological construct that is computed on the basis of these ratings (i.e., metacognitive performance / ability).

Among the five task characteristics of interest (continuous versus discrete rating scales, number of discrete confidence ratings, simultaneous versus sequential type $1 / 2$ responses, feedback, presence of an online staircase), simultaneous type $1 / 2$ responses most strongly benefited the test-retest reliability of $M_{\text {ratio }}$. Compared to post-decisional ratings of confidence, simultaneous type $1 / 2$ responses may be more precise as they are closer to the percept and thus a potential post-decisional memory loss or other sources of noise are minimized. Nevertheless, it is worth noting that one could have made an argument for the reverse finding as well, such that sequential type $1 / 2$ responses give more room for post-stimulus computations of confidence that ultimately lead to more precise and consistent confidence ratings.

Interestingly, contrary to the expectation, there was a weak negative effect of external feedback, i.e. the presence of feedback reduced the test-retest reliability of $M_{\text {ratio }}$. It might be possible that feedback leads to ongoing noisy recalibrations of confidence ratings, which introduces additional variability. However, given the uncertainty of the effect, this finding should be considered as exploratory.

I conclude that studies investigating metacognitive performance should consider the use of simultaneous type 1 and type 2 responses to improve measurement reliability.

\section{Future directions}

What may be ultimately required to isolate type 1 and type 2 performance is a mechanistic model of metacognition that captures relevant sources of metacognitive noise and biases, and describes the functional transformations underlying human reports of confidence. Such a model may allow to reverse-engineer metacognitive noise parameters that characterize metacognitive ability in humans (or different facets thereof) separately from sensory or decisional noise parameters. While there are 
some initial proposals for such models (Bang et al., 2019; Shekhar and Rahnev, 2021), there is currently no established model.

\section{Conclusion}

In sum, this article investigated the type 1 performance dependency and test-retest reliability of metacognitive performance measures. It characterizes the behavior of metacognitive performance measures in dependence of key variables (type 1 performance, overall level of metacognitive noise, number of trials) both on simulated and empirical data. On the basis of these results, it provides guidance and recommendations for researchers interested in investigating metacognitive performance.

\section{Methods and Materials}

\section{Empirical studies}

Analyses of empirical data are based on studies of the Confidence Database, a publicly available repository of dataset with confidence ratings (Rahnev et al., 2020). At the time of access, the database had overall 145 studies. Included studies cover different cognitive domains (e.g. perception, memory or decision making), different confidence scales (such as binary, n-point scales, continuous scales and wagering) collect confidence at different times (for example, after or simultaneous with the decision). Inclusion criteria for studies were tasks with exactly two response options (i.e. excluding studies with more than two or continuous type 1 responses). If not otherwise specified, we included only participants with at least 400 trials and $d^{\prime}>0.5$, which reduced the sample size from $\mathrm{N}=6912$ to $\mathrm{N}=1757$ in these instances.

\section{Metacognitive performance measures}

I evaluated two measures of metacognitive sensitivity ( $A U R O C 2$ and meta- $d^{\prime}$ ) and two measures of metacognitive efficiency ( $M_{\text {ratio }}$ and $M_{\text {diff }}$ ). Meta-d' and its derivatives $M_{\text {ratio }}$ and $M_{\text {diff }}$ was computed on the basis of Python adaptations of the original code as described in (Maniscalco and Lau, 2012, 2014), available at http://www.columbia.edu/ bsm2105/type2sdt/. All code is made available upon the final publication of this article.

Due to the instability of $M_{\text {ratio }}$ at low trial numbers or low levels of type 1 performance (Fleming and Lau, 2014), I evaluated a scenario in which extreme $M_{\text {ratio }}$ values are excluded and three additional regularized variants of $M_{\text {ratio }}$ (Table 1 ). In the first case, referred to as $M_{\text {ratio }}$ (excl.), I excluded participants below and above specified upper bounds. A sensible lower bound is 0 , which corresponds to a metacognitively blind observer. Also note that negative values of $M_{\text {ratio }}$ could show nonsensical behavior. For instance, when meta- $d^{\prime}$ is negative, $M_{\text {ratio }}$ will increase with increasing (positive) type 1 performance, although it should decrease in the logic of a metacognitive performance measure. For the upper bound one might consider 1, which corresponds to an ideal metacognitive observer. However, this will often lead to an asymmetric highly non-normal distribution, as $M_{\text {ratio }}$ values greater than 1 are quite common, possibly also due to post-decisional processing that benefits confidence reports. Instead, here I propose to use an upper bound that is 
symmetric with respect to the mean or median of $M_{\text {ratio }}$ in the Confidence Database (both are around 0.8). In this work I thus use an upper bound of 1.6.

Determining these bounds based on the $M_{\text {ratio }}$ distribution within an individual study will often be problematic, as such estimates are easily skewed by outliers in studies with typical sample sizes. I thus recommend using the bounds 0 and 1.6 proposed here, unless the sample size of a study allows for a sufficiently precise estimate of the distribution.

As a first regularization method, values of $M_{\text {ratio }}$ were simply bounded between the lower and upper bounds explained above. Second, a logarithmic transformation of the $M_{\text {ratio }}$ was tested, which was suggested by (Fleming and Lau, 2014) as a generic regularization method for ratio measures, giving equal weight to increases and decreases relative to an ideal metacognitive observer with $\log \left(M_{\text {ratio }}\right)=0$. Note that taking the logarithm also requires the introduction of a positive lower bound, as the logarithm is undefined in the negative range. Here, 0.1 was chosen as a minimum. Third, a hierarchical Bayesian estimation of $M_{\text {ratio }}$ was evaluated (Fleming, 2017), which effectively tames extreme values by means of a group prior.

Table 1. Variants of $M_{\text {ratio }}$.

\begin{tabular}{|l|l|l|}
\hline Method & Formula & Parameters used in this work \\
\hline $\begin{array}{l}\text { Excluding } \\
\text { Bounding }\end{array}$ & $l b \leq M_{\text {ratio }} \leq u b$ & Lower bound $l b=0$, upper bound $u b=1.6$ \\
\hline $\begin{array}{l}\text { Logarithmic } \\
\text { transformation }\end{array}$ & $\log \max \left(l b, \min \left(u b, M_{\text {ratio }}\right)\right)$ & Lower bound $l b=0$, upper bound $u b=1.6$ \\
\hline $\begin{array}{l}\text { Hierarchical } \\
\text { estimation }\end{array}$ & see Fleming $(2017)$ & Lower bound $l b=0.1$ \\
\hline
\end{tabular}

\section{Measures of test-retest reliability}

The test-retest reliability of metacognitive performance measures was evaluated both by means of the Pearson correlation and by means of a measure of absolute error, the normalized mean absolute error (NMAE). While the Pearson correlation is scale- and mean-invariant and thus quantifies to what degree the pattern of results is similar across participants between two experimental sessions, the NMAE takes into account possible mean or scale shifts.

Let $\mathbf{x}$ and $\mathbf{y}$ be two vectors containing the metacognitive performance values of the $\mathrm{N}$ subjects for a test session $(\mathbf{x})$ and a retest session $(\mathbf{y})$. The Pearson reliability is computed as the standard sample correlation coefficient between paired data $\mathbf{x}$ and $\mathbf{y}$. The NMAE of vectors $\mathbf{x}$ and $\mathbf{y}$ of length $\mathrm{N}$ is defined as follows:

$$
\operatorname{NMAE}(\mathbf{x}, \mathbf{y})=\frac{1}{N} \sum_{i=1}^{N}\left|x_{i}-y_{i}\right| /\left(\frac{1}{2 N} \sum_{i=1}^{N}\left|x_{i}-\bar{y}\right|+\frac{1}{2 N} \sum_{i=1}^{N}\left|y_{i}-\bar{x}\right|\right)
$$


While the numerator is the regular mean absolute error, the denominator is the mean absolute difference of each value and the average of the other session. The denominator ensures that the overall error is largely independent of the scaling of a measure, thus allowing comparisons between different metacognitive performance measures.

Note that the NMAE is not valid for the hierarchical $M_{\text {ratio }}$ and was thus not computed for this metacognitive performance measure. The reason is that separate group priors are applied to the data of the test and retest set, which bias individual values to the respective group mean. The resulting distributions for test and retest have often considerable mean differences with individual values being tightly clustered around the respective means. While the overall mean difference is normalized for (denominator of the NMAE), the compression around the distribution means will lead to an artifactual reduction of the NMAE.

\section{Simulations}

\subsection{General model}

I simulated artificial observers that were presented with stimuli pertaining to two stimulus categories. The task of observers was to identify the correct stimulus category, i.e., a binary choice task. I assume that percepts are subject to Gaussian sensory noise with standard deviation $\sigma_{s}$ :

$$
\text { percept } \sim \mathcal{N}\left( \pm \frac{\mu}{2}, \sigma_{\mathrm{s}}\right)
$$

where $\pm \mu / 2$ are the stimulus means.

Using Bayes' rule, the observer computes the choice probability $p$ as follows:

$$
p=\frac{1}{1+\exp \left(-\frac{\mu \cdot p e r c e p t}{\sigma_{\mathrm{s}}^{2}}\right)}
$$

The observer chooses stimulus category 2 whenever $p \geq 0.5$ and else stimulus category 1 . An auxiliary (choice-independent) confidence variable $c \in[0 ; 1]$ is computed from this choice probability:

$$
c= \begin{cases}2 \cdot(0.5-p) & \text { if } p<0.5 \\ 2 \cdot(p-0.5) & \text { else }\end{cases}
$$

However, the final confidence reports are subject to metacognitive noise described by a metacognitive noise distribution $\mathcal{M}$ with mode $c$ and metacognitive noise $\sigma_{\mathrm{m}}$ :

$$
\text { confidence } \sim \mathcal{M}\left(c, \sigma_{\mathrm{m}}\right)
$$

The parameter $\sigma_{\mathrm{m}}$ of the metacognitive noise distribution $\mathcal{M}$ defines the spread of the distribution. For the simulations in this work, the Beta distribution was used, which is naturally bounded between 0 and 1 :

$$
\text { confidence } \sim \frac{1}{B(\alpha, \beta)} x^{\alpha-1}(1-x)^{\beta-1}
$$


The used parameterization is $\alpha=c\left(1 / \sigma_{m}-2\right)+1$ and $\beta=(1-c)\left(1 / \sigma_{m}-2\right)+1$, for which the Beta distribution has mode $c$ and spread $0<\sigma_{\mathrm{m}} \leq 0.5$ (note that $\sigma_{\mathrm{m}}$ is not a standard deviation). For the maximum value of $\sigma_{\mathrm{m}}=0.5$, the Beta distribution becomes the uniform distribution.

\subsection{The relationship between type 1 performance and metacognitive performance}

To assess the relationship between type 1 performance and metacognitive performance measures, type 1 and confidence data were simulated for different type 1 and 2 performance levels. One hundred different performance levels were evaluated by varying the sensory noise parameter $\sigma_{s}$ from 0 (no sensory noise, i.e. perfect performance) and 5 (very high sensory noise, approximately chance-level performance). Six metacognitive noise levels were assessed by varying $\sigma_{\mathrm{m}}$ between 0 and 0.5 in steps of 0.1 . For each pair $\left(\sigma_{s}, \sigma_{m}\right), 1000$ subjects with 10,000 trials each were simulated.

\subsection{Test-retest reliability}

To evaluate the test-retest reliability of metacognitive performance measures, two experimental sessions were simulated for each subject. Type 1 performance and the number of trials in a session are expected to be critical factors for the test-retest reliability and were hence under special consideration. Specifically, the sensory noise parameter $\sigma_{\mathrm{s}}$ was varied to induce different levels of type 1 performance between 55\% correct and 95\% correct and the number of trials was varied between 25 and 1000 in steps of 25. For each pair ( $\sigma_{s}$, \#trials), two independent experimental sessions of 100 subjects were generated. For each session, all metacognitive performance measures under consideration were computed. Reliability was assessed both by means of the Pearson correlation and the NMAE.

To estimate uncertainty, the entire procedure described above was repeated 300 times with different random seeds.

\section{Analyses of empirical data}

\subsection{Preprocessing}

All studies were transformed to a homogenous format. Most importantly, as the computation of meta- $d$ ' requires discrete confidence ratings, a discretization procedure was defined. The maximum number of discrete confidence ratings was limited to 6 , as this is the highest number that was used in a major proportion of studies. Trials were sorted into six bins on a percentile basis (aiming for a balanced number in all confidence bins) with the constraint that all bins must be unique. Negative confidence ratings, which some studies included to indicate that one is confident in being wrong, were floored at 1 , the lowest confidence rating. Verbal confidence ratings were converted to appropriate numerical ratings (e.g. "difficult" and "easy" were converted to ratings 1 and 2) and fractional ratings (e.g., indicating the probability of being correct) were converted to integers. While it is clear that there is uncertainty in how to convert different confidence rating procedures to a common scale, this is an issue inherent to any particular scale already at the response stage, as there is generally little knowledge of how participants translate perceived levels of confidence to available rating options. 


\subsection{The relationship between type 1 performance and metacognitive performance}

The relationship between $d^{\prime}$ and metacognitive performance measures in the Confidence Database was characterized in two ways. First, the overall linear slope between type 1 and type 2 performance across all subjects was computed by means of ordinary least square regression. This analysis shows whether there is a systematic linear trend between type 1 performance and each metacognitive performance measure. To derive a more fine-grained empirical function, averages for $d^{\prime}$ bins were computed, centered at values ranging from 0.3 to 2.9 in steps of 0.2 (window size: \pm 0.2 ) and standard errors within each bin were computed. The $d^{\prime}$ range was limited to a range of 0.1 to 3.1 (only a few subjects show even smaller or greater type 1 performance.

\subsection{Test-retest reliability}

As it is possible that the test-retest reliability of metacognitive performance measures strongly depends on idiosyncratic characteristics of empirical data, I also evaluated the test-retest reliability of studies in the Confidence Database. As the number of trials per subject is a strong predictor of test-retest reliability, the studies of the Confidence Database were divided according to the split-half number of trials ( 5 bins: 0-200 trials, ..., 800-1000 trials). For each subject, every second trial was assigned to the "test session" and every other trial to the "retest session". As in the simulation, I computed both the Pearson correlation coefficient and the normalized mean absolute error (NMAE; Eq. 1) as measures of reliability.

\section{Acknowledgements}

This research was funded by the German Research Foundation (grant GU 1845/1-1). I'm grateful to the lab of Elisa Filevich for critical discussion. Computation has been performed on the HPC for Research cluster of the Berlin Institute of Health.

\section{Data and code availability}

The Confidence Database (Rahnev et al., 2020) is available at https://osf.io/s46pr. Code for all analyses and simulations is available under https://github.com/m-guggenmos/metameasure.

\section{References}

Bahrami B, Olsen K, Bang D, Roepstorff A, Rees G, Frith C (2012) What failure in collective decision-making tells us about metacognition. Philos Trans R Soc B Biol Sci 367:1350-1365.

Baird B, Cieslak M, Smallwood J, Grafton ST, Schooler JW (2015) Regional White Matter Variation Associated with Domain-specific Metacognitive Accuracy. J Cogn Neurosci 27:440-452.

Baird B, Mrazek MD, Phillips DT, Schooler JW (2014) Domain-specific enhancement of metacognitive ability following meditation training. J Exp Psychol Gen 143:1972-1979.

Baird B, Smallwood J, Gorgolewski KJ, Margulies DS (2013) Medial and Lateral Networks in Anterior 
Prefrontal Cortex Support Metacognitive Ability for Memory and Perception. J Neurosci 33:16657-16665.

Bang JW, Shekhar M, Rahnev D (2019) Sensory noise increases metacognitive efficiency. J Exp Psychol Gen 148:437-452.

Carroll JB (1993) Human Cognitive Abilities: A Survey of Factor-Analytic Studies. Cambridge: Cambridge University Press. Available at:

https://www.cambridge.org/core/books/human-cognitive-abilities/F83D5EADF14A453F6350 FF3DD39631C8 [Accessed September 16, 2021].

Clarke FR, Birdsall TG, Tanner WP (1959) Two Types of ROC Curves and Definitions of Parameters. J Acoust Soc Am 31:629-630.

Critchfield TS (1993) Signal-detection properties of verbal self-reports. J Exp Anal Behav 60:495-514.

Faivre N, Roger M, Pereira M, de Gardelle V, Vergnaud J-C, Passerieux C, Roux P (2020) Confidence in visual motion discrimination is preserved in individuals with schizophrenia. J Psychiatry Neurosci 45:200022.

Filevich E, Koß C, Faivre N (2020) Response-Related Signals Increase Confidence But Not Metacognitive Performance. eneuro 7:ENEURO.0326-19.2020.

Fitzgerald LM, Arvaneh M, Dockree PM (2017) Domain-specific and domain-general processes underlying metacognitive judgments. Conscious Cogn 49:264-277.

Fleming SM (2017) HMeta-d : hierarchical Bayesian estimation of metacognitive efficiency from confidence ratings. Neurosci Conscious 2017:1-14.

Fleming SM, Dolan RJ, Frith CD (2012) Metacognition: computation, biology and function. Philos Trans R Soc B Biol Sci 367:1280-1286.

Fleming SM, Lau H (2014) How to measure metacognition. Front Hum Neurosci 8:443.

Frith C (2008) Social cognition. Philos Trans R Soc B Biol Sci 363:2033-2039.

Galvin SJ, Podd JV, Drga V, Whitmore J (2003) Type 2 tasks in the theory of signal detectability: Discrimination between correct and incorrect decisions. Psychon Bull Rev 10:843-876.

García-Pérez MA (1998) Forced-choice staircases with fixed step sizes: asymptotic and small-sample properties. Vision Res 38:1861-1881.

Harrison OK, Garfinkel SN, Marlow L, Finnegan S, Marino S, Nanz L, Allen M, Finnemann J, Keur-Huizinga L, Harrison SJ, Stephan KE, Pattinson KTS, Fleming SM (2020) The Filter Detection Task for measurement of breathing-related interoception and metacognition. Neuroscience. Available at: http://biorxiv.org/lookup/doi/10.1101/2020.06.29.176941 [Accessed September 2, 2021].

Hauser TU, Allen M, NSPN Consortium, Rees G, Dolan RJ (2017) Metacognitive impairments extend perceptual decision making weaknesses in compulsivity. Sci Rep 7:6614.

Hertz U, Bell V, Barnby J, McQuillin A, Bahrami B (2020) The communication of metacognition for social strategy in psychosis: an exploratory study. Schizophr Bull Open.

Hosseini J, Ferrell WR (1982) Detectability of correctness: A measure of knowing that one knows. Instr Sci 11:113-127.

Jansen RA, Rafferty AN, Griffiths TL (2021) A rational model of the Dunning-Kruger effect supports insensitivity to evidence in low performers. Nat Hum Behav 5:756-763.

Konishi M, Compain C, Berberian B, Sackur J, de Gardelle V (2020) Resilience of perceptual metacognition in a dual-task paradigm. Psychon Bull Rev Available at: http://link.springer.com/10.3758/s13423-020-01779-8 [Accessed November 24, 2020].

Lee ALF, Ruby E, Giles N, Lau H (2018) Cross-Domain Association in Metacognitive Efficiency Depends on First-Order Task Types. Front Psychol 9:2464.

Maniscalco B, Lau H (2012) A signal detection theoretic approach for estimating metacognitive sensitivity from confidence ratings. Conscious Cogn 21:422-430.

Maniscalco B, Lau H (2014) Signal Detection Theory Analysis of Type 1 and Type 2 Data: Meta-d0, Response- Specific Meta-d0, and the Unequal Variance SDT Model. In: The cognitive neuroscience of metacognition (Fleming SM, Frith CD, eds), pp 25-66. Basel: Springer-Verlag 
Publishing. Available at: https://doi.org/10.1007/978-3-642-45190-4_3.

Maniscalco B, Lau H (2015) Manipulation of working memory contents selectively impairs metacognitive sensitivity in a concurrent visual discrimination task. Neurosci Conscious 2015 Available at: https://academic.oup.com/nc/article/doi/10.1093/nc/niv002/2757110 [Accessed November 24, 2020].

Mazor M, Fleming SM (2021) The Dunning-Kruger effect revisited. Nat Hum Behav 5:677-678.

McCurdy LY, Maniscalco B, Metcalfe J, Liu KY, de Lange FP, Lau H (2013) Anatomical Coupling between Distinct Metacognitive Systems for Memory and Visual Perception. J Neurosci 33:1897-1906.

Mei N, Rankine S, Olafsson E, Soto D (2020) Similar history biases for distinct prospective decisions of self-performance. Sci Rep 10:5854.

Nelson TO (1984) A Comparison of Current Measures of the Accuracy of Feeling-of-Knowing Predictions. Psychol Bull 95:109-133.

Nicholson T, Williams DM, Lind SE, Grainger C, Carruthers P (2020) Linking metacognition and mindreading: Evidence from autism and dual-task investigations. J Exp Psychol Gen Available at: http://doi.apa.org/getdoi.cfm?doi=10.1037/xge0000878 [Accessed November 24, 2020].

Odegaard B, Chang MY, Lau H, Cheung S-H (2018) Inflation versus filling-in: why we feel we see more than we actually do in peripheral vision. Philos Trans R Soc B Biol Sci 373:20170345.

Ordin M, Polyanskaya L (2020) The role of metacognition in recognition of the content of statistical learning. Psychon Bull Rev.

Ordin M, Polyanskaya L, Soto D (2020) Metacognitive processing in language learning tasks is affected by bilingualism. J Exp Psychol Learn Mem Cogn 46:529-538.

Pollack I (1959) On Indices of Signal and Response Discriminability. J Acoust Soc Am 31:1031-1031.

Rahnev D et al. (2020) The Confidence Database. Nat Hum Behav.

Rahnev D, Fleming SM (2019) How experimental procedures influence estimates of metacognitive ability. Neurosci Conscious 5:1-9.

Reyes G, Vivanco-Carlevari A, Medina F, Manosalva C, de Gardelle V, Sackur J, Silva JR (2020) Hydrocortisone decreases metacognitive efficiency independent of perceived stress. Sci Rep 10:14100.

Rouault M, Seow T, Gillan CM, Fleming SM (2018) Psychiatric Symptom Dimensions Are Associated With Dissociable Shifts in Metacognition but Not Task Performance. Biol Psychiatry 84:443-451.

Rounis E, Maniscalco B, Rothwell JC, Passingham RE, Lau H (2010) Theta-burst transcranial magnetic stimulation to the prefrontal cortex impairs metacognitive visual awareness. Cogn Neurosci 1:165-175.

Sadeghi S, Ekhtiari H, Bahrami B, Ahmadabadi MN (2017) Metacognitive Deficiency in a Perceptual but Not a Memory Task in Methadone Maintenance Patients. Sci Rep 7:7052.

Samaha J, Postle BR (2017) Correlated individual differences suggest a common mechanism underlying metacognition in visual perception and visual short-term memory. Proc R Soc B Biol Sci 284:10.

Shapiro SS, Wilk MB (1965) An analysis of variance test for normality (complete samples). Biometrika 52:591-611.

Shekhar M, Rahnev D (2018) Distinguishing the Roles of Dorsolateral and Anterior PFC in Visual Metacognition. J Neurosci 38:5078-5087.

Shekhar M, Rahnev D (2021) The nature of metacognitive inefficiency in perceptual decision making. Psychol Rev 128:45-70.

Ye Q, Zou F, Dayan M, Lau H, Hu Y, Kwok SC (2019) Individual susceptibility to TMS affirms the precuneal role in meta-memory upon recollection. Brain Struct Funct 224:2407-2419. 


\title{
Supplement to: Measuring metacognitive performance: type 1 performance dependence and test-retest reliability
}

\begin{abstract}
Matthias Guggenmos ${ }^{1 *}$
${ }^{1}$ Charité - Universitätsmedizin Berlin, corporate member of Freie Universität Berlin and Humboldt-Universität zu Berlin, Department of Psychiatry and Neurosciences, Charitéplatz 1, 10117 Berlin, Germany
\end{abstract}

* Corresponding author: Matthias Guggenmos (mg.corresponding@gmail.com) 


\section{Supplementary Figure S1. Simulation: test-retest reliability of additional}

\section{metacognitive performance measures}

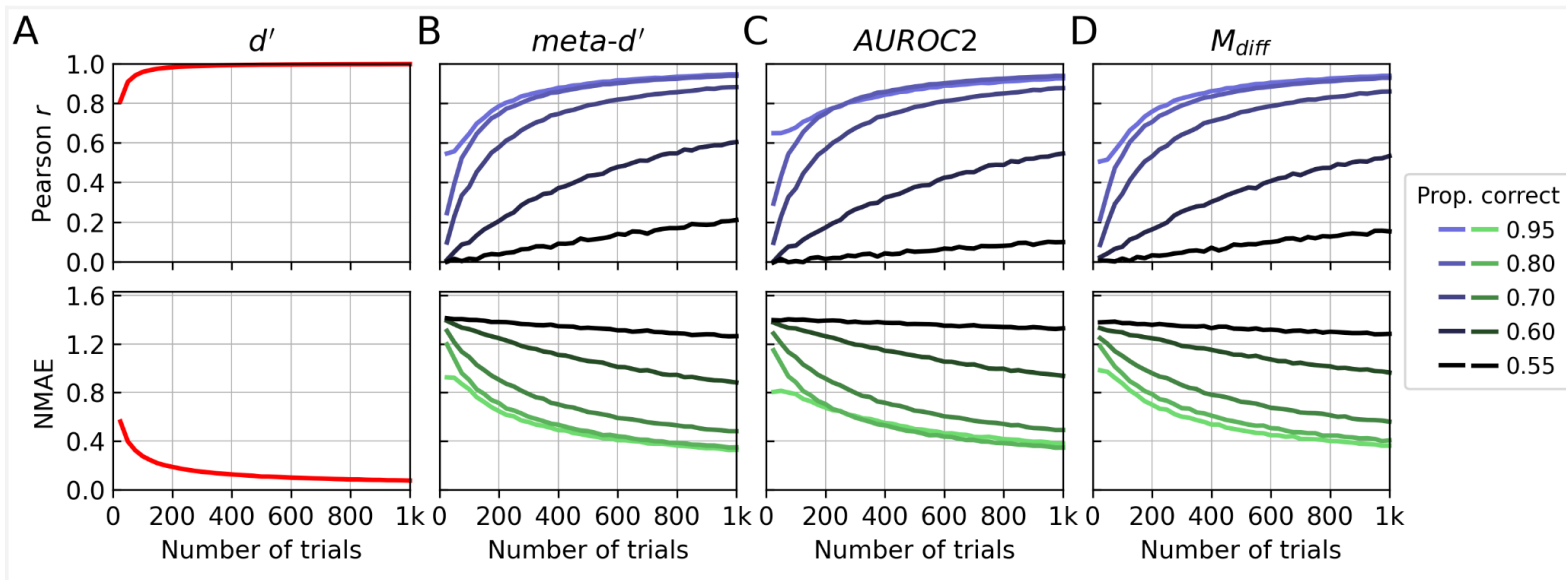

Supplementary Figure S1. Simulation: test-retest reliability of type 1 performance and additional metacognitive performance measures. Note that in contrast to metacognitive performance measures, the reliability of $d^{\prime}$ was computed across the entire range of simulated type 1 performance levels. 


\section{Supplementary Figure S2. Simulation: differences in test-retest reliability of}

\section{regularized $M_{\text {ratio }}$ variants}
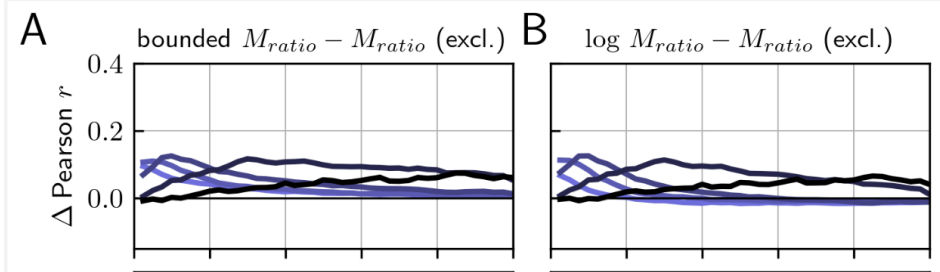

C bounded $M_{\text {ratio }}-\log M_{\text {ratio }}$
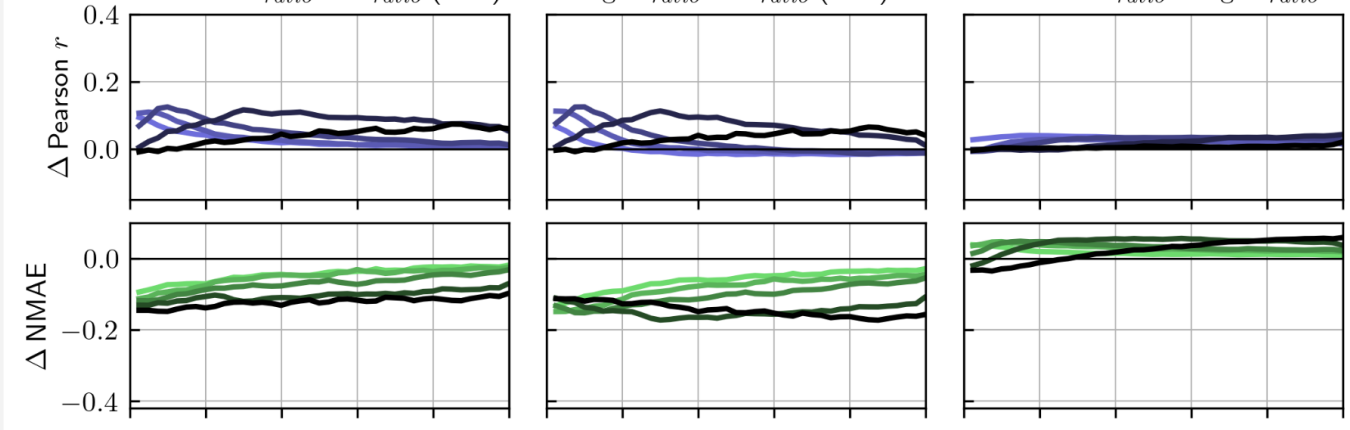

Proportion

correct

$-0.95$

$-0.80$

$-0.70$

$-0.60$

$-0.55$

D hierarchical $M_{\text {ratio }}-$ bounded $M_{\text {ratio }} \mathrm{E}$ hierarchical $M_{\text {ratio }}-\log M_{\text {ratio }} \mathrm{F}_{\text {hierarchical }} M_{\text {ratio }}-M_{\text {ratio }}$ (excl.)

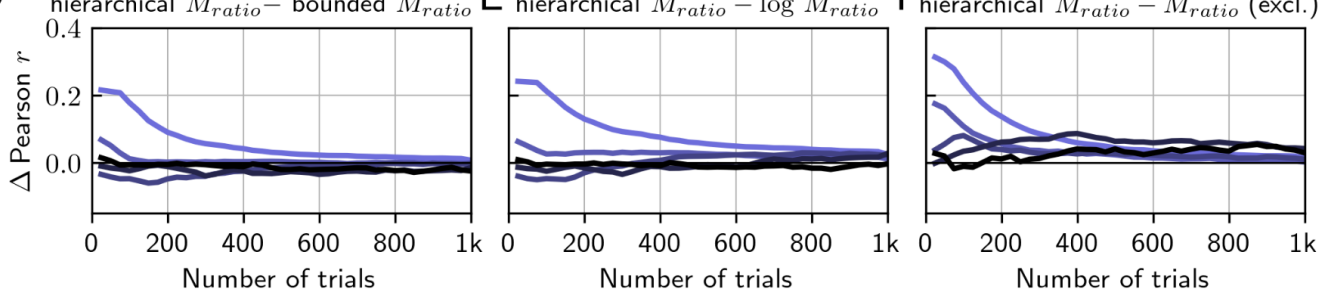

Supplementary Figure S2. Simulation: differences in test-retest reliability of $\boldsymbol{M}_{\text {ratio }}$ variants. Note that the NMAE is not valid for the hierarchical Mratio (see method section 3) and is thus not shown. 
Supplementary Figure S3. Empirical test-retest reliability of type 1 performance and additional metacognitive performance measures

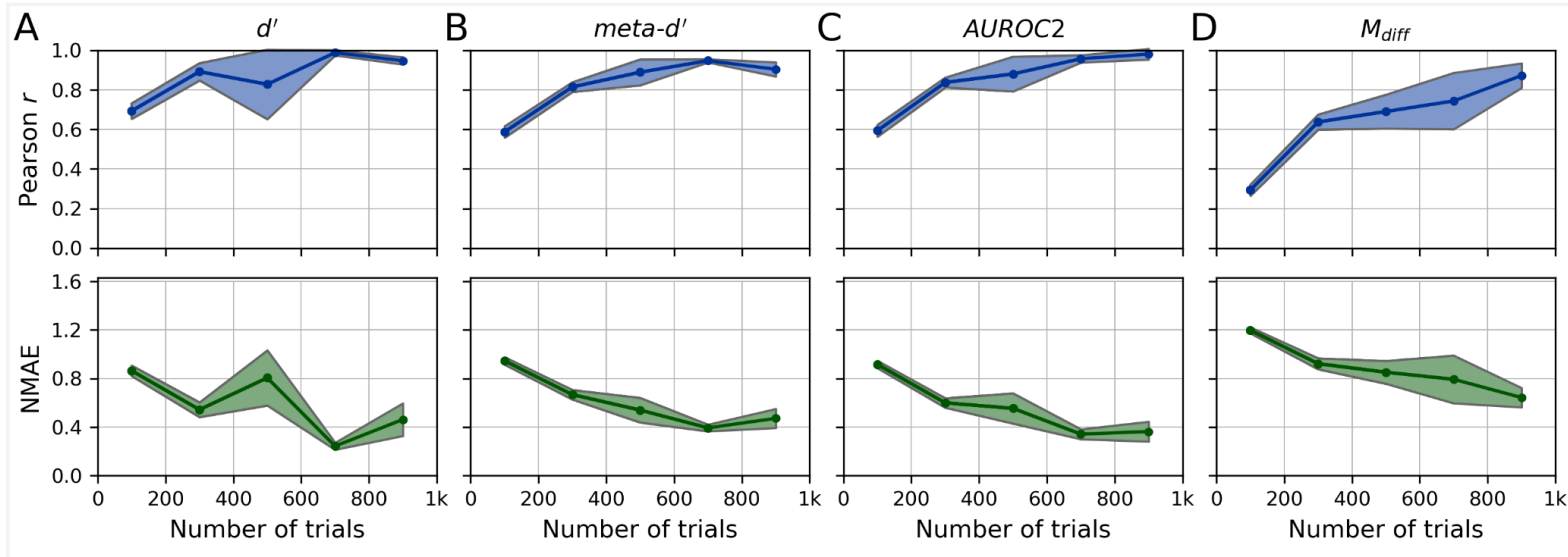

Supplementary Figure S3. Empirical test-retest reliability of type 1 performance and additional metacognitive performance measures. Test-retest reliability is computed either as the Pearson correlation coefficient or the normalized mean absolute error (NMAE) between test and retest trials. The $x$-axis denotes the average split-half number of trials for the Confidence Database studies (bins of 200 from 0-1000 trials), i.e. the number of trials per test and retest. In this way, the expected reliability for other/new studies with overall $\mathrm{N}$ trials can simply be obtained by looking up the reliability for $\mathrm{x}=\mathrm{N}$. Shaded areas indicate standard errors across studies. 


\section{Supplementary Figure S4. Type 1 performance dependency of $M_{\text {ratio }}$ for other metacognitive noise distributions}
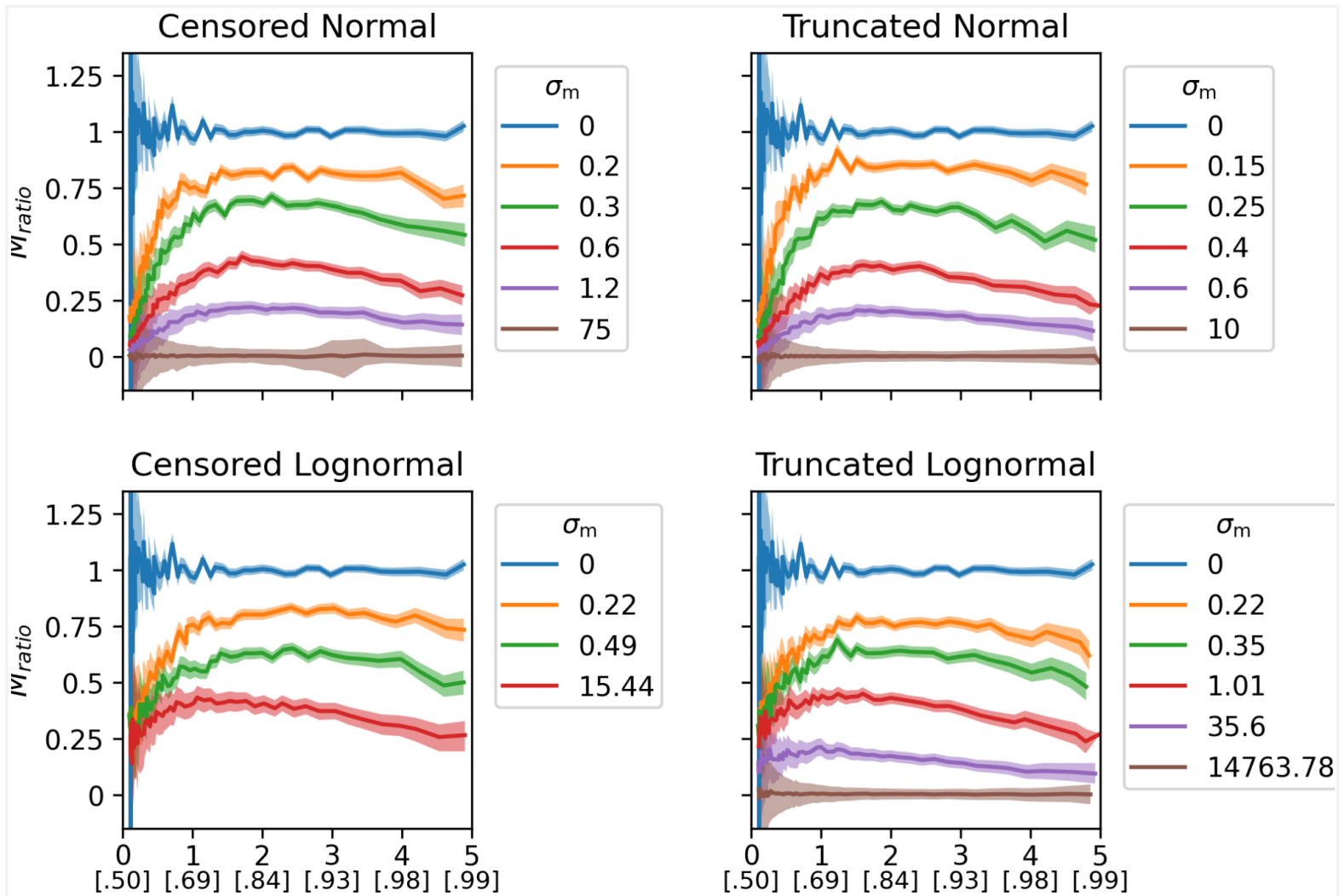

Type 1 performance d' [proportion correct]

Supplementary Figure S4. Type 1 performance dependency of $\boldsymbol{M}_{\text {ratio }}$ for other metacognitive noise distributions. Normal and lognormal noise distributions were constricted to the range $[0 ; 1]$ either via truncation or censoring. Truncating a distribution means to cut off the parts of the distribution outside the truncation points (here confidence $<0$ and confidence $>1$ ) and to renormalize the remainder of the distribution to 1 . In the case of censoring, all values below 0 and above 1 are set to 0 and 1 , respectively. The values for the parameter $\sigma_{\mathrm{m}}$ were chosen such that the average $M_{\text {ratio }}$ values at each metacognitive noise level approximately match the average $M_{\text {ratio }}$ values of the original Beta noise distribution. For the censored lognormal distribution, very high levels of metacognitive noise generate only maximal confidence ratings and are thus omitted. Shaded areas denote asymmetrical standard deviations. 